\title{
In Planta Colonization and Role of T6SS in Two Rice Kosakonia Endophytes
}

\author{
Susan Mosquito, ${ }^{1}$ Iris Bertani, ${ }^{1}$ Danilo Licastro, ${ }^{2}$ Stéphane Compant, ${ }^{3}$ Michael P. Myers, ${ }^{1}$ \\ Estefanía Hinarejos, ${ }^{4}$ Asaf Levy, ${ }^{5}$ and Vittorio Venturi ${ }^{1,+}$ \\ ${ }^{1}$ International Centre for Genetic Engineering and Biotechnology, 34149 Trieste, Italy \\ ${ }^{2}$ CBM S.c.r.l., Area Science Park-Basovizza, 34149 Trieste, Italy \\ ${ }^{3}$ Bioresources Unit, Center for Health \& Bioresources, AIT Austrian Institute of Technology GmbH, 3430 Tulln, Vienna, Austria \\ ${ }^{4}$ Microbial Active Technical Experts, Valencia, Spain \\ ${ }^{5}$ Department of Plant Pathology and Microbiology, The Robert H. Smith Faculty of Agriculture, Food, and Environment, The \\ Hebrew University of Jerusalem, Rehovot 76100, Israel
}

Accepted 10 October 2019.

\begin{abstract}
Endophytes live inside plants and are often beneficial. Kosakonia is a novel bacterial genus that includes many diazotrophic plant-associated isolates. Plant-bacteria studies on two rice endophytic Kosakonia beneficial strains were performed, including comparative genomics, secretome profiling, in planta tests, and a field release trial. The strains are efficient rhizoplane and root endosphere colonizers and localized in the root cortex. Secretomics revealed 144 putative secreted proteins, including type VI secretory system (T6SS) proteins. A Kosakonia T6SS genomic knock-out mutant showed a significant decrease in rhizoplane and endosphere colonization ability. A field trial using rice seed inoculated with Kosakonia spp. showed no effect on plant growth promotion upon nitrogen stress and microbiome studies revealed that Kosakonia spp. were significantly more present in the inoculated rice. Comparative genomics indicated that several protein domains were enriched in plant-associated Kosakonia spp. This study highlights that Kosakonia is an important, recently classified genus involved in plant-bacteria interaction.
\end{abstract}

Keywords: endophytes, genomics, microscopy and imaging

Rice is the most important food crop in the developing world, being a staple food for over two billion people in Asia and for many millions in Africa and Latin America (Khush 2003; Zeigler and Barclay 2008). The challenge in the future will be to increase rice yields for a growing world population and decrease the use of chemical pesticides and fertilizers for more sustainable approaches (Mano and Morisaki 2008; Schütz et al. 2018). The use of microbially based biopesticides and biofertilizers is currently believed to be a promising way to render agriculture more sustainable by reducing the chemical input

${ }^{\dagger}$ Corresponding author: V. Venturi; vittorio.venturi@icgeb.org

Funding: S. Mosquito is the beneficiary of a fellowship from the International Centre for Genetic Engineering and Biotechnology.

*The $\boldsymbol{e}$-Xtra logo stands for "electronic extra" and indicates that seven supplementary figures and six supplementary tables are published online.

The author(s) declare no conflict of interest.

๑) 2020 The American Phytopathological Society
(Berg 2009; Gupta and Dikshit 2010; Mahanty et al. 2017; Schütz et al. 2018).

Plant-associated microbiota constitute the plant microbiome playing a fundamental role in plant growth promotion (PGP) and health (Okubo et al. 2014; Schlaeppi and Bulgarelli 2015; Turner et al. 2013). PGP activities by plant-associated microbes include induction of plant immunity, acquisition of nutrients, and resistance to biotic or abiotic stresses (Compant et al. 2010; Glick 2014; Lugtenberg and Kamilova 2009). The plant microbiome represents many diverse microorganisms that interact and colonize different plant-associated niches (Müller et al. 2016; van der Heijden and Hartmann 2016). One of these compartments is the rhizoplane, which consists of the rootsurrounding soil being influenced by root exudates and has a high diversity and distribution of microbial life (Berg et al. 2005; Hartmann et al. 2008; Lundberg et al. 2012). Some microbiome members not only colonize the rhizoplane but also thrive as endophytes inside plant tissues (Berg et al. 2014; Reinhold-Hurek and Hurek 2011). Endophytes mostly enter via the roots and have evolved an intimate relationship with the plant host; many endophytes do not elicit a plant immune response and some display PGP properties (Garrido-Oter et al. 2018; Glick 2014; Hayat et al. 2010; Reinhold-Hurek and Hurek 1998; Sessitsch et al. 2012). Therefore, endophytes constitute an important class of beneficial bacteria now considered to be a potentially important group that can be used as microbial inoculants for a more sustainable agriculture. However, more information is needed about the endophytic life style and mechanisms of plant entry and colonization.

General features of the rice microbiome, and plant microbiomes in general, include less species richness in the plant endosphere than on the rhizoplane (surface of the root) and in the rhizosphere (Bulgarelli et al. 2012; Edwards et al. 2015; Lundberg et al. 2012). Microbial communities of the rice rhizoplane and root endosphere stabilize after 7 to 8 weeks from germination due to the plant life cycle (Edwards et al. 2017). The rice endophytic bacteriome has a prevalence of Proteobacteria, representing more than $50 \%$ of the bacterial community, with $\gamma$-proteobacteria being the most abundant class. Many rice endophytes possess nitrogen fixation genes as well as genes related to nitrification and denitrification processes, which suggest that they are involved in the entire nitrogen cycle (Sessitsch et al. 2012). Examples of PGP rice endophytes include Pantoea agglomerans YS19 (Feng et al. 2006; Jiang et al. 2015; Yang et al. 1999) and Pseudomonas stutzeri A15, a 
rhizospheric and endospheric diazotrophic root colonizer (Pham et al. 2017). Other rice endophytes with potential use as nitrogen biofertilizers include Gluconacetobacter diazotrophicus LMG7603, Herbaspirillum seropedicae LMG6513, Azospirillum lipoferum 4B (LMG4348), and Burkholderia vietnamiensis LMG10929 (Baldani et al. 1986; Govindarajan et al. 2008; Rouws et al. 2010; Trân Van et al. 1996).

We previously reported the isolation and characterization of bacterial endophytes from rice grown in Italy that resulted in a collection of more than 1,300 putative isolates (Bertani et al. 2016). Several in vitro and in planta selection steps resulted in a smaller set of putative endophytes, which displayed efficient in planta colonization levels as well as having PGP traits. Among these were two strains that belong to the recently described Kosakonia genus (Alnajar and Gupta 2017; Brady et al. 2013), which consists mostly of plant-associated diazotrophs (Kämpfer et al. 2016; Li et al. 2017). Some Kosakonia strains (for example, Kosakonia radicincitans DSM 16656) are promiscuous endophytes and promote plant growth in different plants, including wheat, maize, tomato, pea, and cruciferous vegetables (Berger et al. 2013; Höflich and Ruppel 1994; Schreiner et al. 2009). In recent years, members of this genus have gained attention and several genome sequences have been reported (Becker et al. 1997; Bergottini et al. 2015; Chen et al. 2014; Kämpfer et al. 2016; Li et al. 2017; Mohd Suhaimi et al. 2014; Shinjo et al. 2016).

In this study, we characterized two Kosakonia strains that we previously isolated as PGP endophytes of rice (Bertani et al. 2016). In order to begin to study features that make them efficient endophytic colonizers, we performed plant colonization, genomic, and protein secretome studies. We also report a rice field release study of one Kosakonia strain and its effect on plant yield and on the rice root endophytic microbiome.

\section{RESULTS}

\section{Genome sequence and analysis of the two Kosakonia strains.}

It was of interest to determine the genome sequences of the two rice-beneficial Kosakonia sp. endophytic strains KO348 and KO774, which we previously identified (Bertani et al. 2016). We previously reported the genome sequence of strain KO348 (Meng et al. 2015) and in this study we resequenced it; the assembly yielded a higher-quality genome compared with the previous version. The new sequence gave 26 scaffolds with an average size of $192.7 \mathrm{kbp}$ versus 56 scaffolds with an average scaffold size of $89.3 \mathrm{kbp}$ in the new versus the old genome version, respectively. In the case of strain KO774, we report here for the first time the genome sequence, which was performed on an Illumina MiSeq platform $(2 \times 300 \mathrm{bp})$ by de novo assembly using Velvet 1.2.09. The assembly using the Integrate Microbial Genomes and Microbiomes (IMG/M) database (Kosakonia sp. KO774) yielded 12 scaffolds giving a total of 4,875,574 bp, including 4,530 putative protein-coding genes and 153 RNA genes; the assembly also revealed a putative plasmid (74 kbp). The contig containing the putative plasmid included loci for plasmid replication, conjugation, segregation genes, a few toxin-antitoxin systems, and a large cellobiose phosphorylase gene (Supplementary Fig. S1).

The bacterial genomes KO348 and KO774 had 3,853 orthologous genes identified as bidirectional best hits $(\mathrm{BBH})$; namely, $82.5 \%$ of the genes in each genome displayed at least $70 \%$ sequence identity over at least $70 \%$ of the length of the shorter sequence in each BBH pair. The genomic average nucleotide identity between the BBH pairs was $83.69 \%$ (Varghese et al. 2015). Both strains share some PGP-related genes involved in siderophore production (enterobactins), phosphate solubilization (phytase), flagellar motility, plant tissue degrading enzymes (cellulase), and the nif gene cluster for nitrogen fixation. The genome size of KO774 is approximately $100 \mathrm{~kb}$ smaller compared with the one of KO348. Interestingly, the KO348 strain has extra phage-related proteins whereas KO774 has a higher copy number of flagellin-related proteins (Supplementary Table S1).

\section{Comparative genomics in the Kosakonia genus.}

A phylogenetic tree was constructed comprising all of the Kosakonia complete genomes publicly available in the IMG/M database of the Joint Genome Institute (Nordberg et al. 2014), including strains $\mathrm{KO} 348$ and $\mathrm{KO} 774(n=15)$; the analysis was performed having Escherichia coli K12 MG1655 as an outgroup. The phylogenetic analysis showed that the strain Kosakonia sp. KO348 is most closely related to the strain K. sacchari CGMCC.1.12101, while Kosakonia sp. KO774 is an outgroup distantly related to the phytophyla group (Fig. 1). The hierarchical clustering in the phylogenetic tree showed a clear separation between two groups: the Kosakonia strains isolated from plants (phytophyla group) and the Kosakonia isolated from human or animal samples (Fig. 1). Interestingly, when analyzing the enriched protein domains between the genomes of the phytophyla group and the human- or animalassociated group, we found that, in the Kosakonia phytophyla group, nitrogen fixation, cobalamin biosynthesis, ethanolamine, and phosphonate metabolism domains were enriched whereas, in the human- or animal-associated strains, the domains for host adaptation and virulence such as immunoglobulin A1 protease and Haem utilization were enriched (Fig. 1; Supplementary Table S2).

\section{Plant colonization assays on rhizoplane and endosphere.}

To determine the colonization ability of the rice rhizoplane and endosphere of both Kosakonia strains and also determine whether they out-competed each other, we conducted colonization studies. Single-inoculation and coinoculation using both Kosakonia strains were performed (Fig. 2). Both Kosakonia sp. strains KO348 and KO774 were able to colonize the rice rhizoplane and endosphere efficiently and at very similar levels when inoculated independently; no statistically significant differences were found between the colonization abilities of both strains (Fig. 2). When both strains were coinoculated on rice, both were able to equally colonize the two plant compartments; no statistically significant differences were found between the Kosakonia strains, without out-competing each other and likely forming stable and mixed communities.

Bacterial CFU of strain KO348 attached to the surface of the seedling root after $1 \mathrm{~h}$ of inoculation was $1.2 \times 10^{7} \mathrm{CFU} / \mathrm{g}$ of root and the number of bacterial cells recovered from the root endosphere after 30 days postinoculation (dpi) was, on average, $1.8 \times 10^{4} \mathrm{CFU} / \mathrm{g}$ of root (Fig. 3), which indicates that KO348 is a good endophytic root colonizer. We also determined the root endosphere colonization at three other time points: 5,10 , and 50 dpi showed $1.8 \times 10^{4}, 8.3 \times 10^{4}$, and $1.1 \times 10^{4} \mathrm{CFU} / \mathrm{g}$ of root, respectively. These latter experiments are the results at each time point of five plants handled and processed independently. It was concluded that Kosakonia sp. KO348 is a good and stable rice root endosphere colonizer under the tested conditions.

\section{Visualization by confocal microscopy in the rice} rhizoplane and endosphere of Kosakonia sp. strain KO348.

In order to unequivocally determine the internal plant colonization by Kosakonia sp. KO348, confocal microscopy localization was performed. Location of the strain on the rhizoplane (including root hair zone, secondary root emergence, and grain surface) and inside the root endosphere 
(transversal sections of the root) of rice roots were determined within rice plants inoculated with Kosakonia strain KO348(pBBRgfp) at different time points $(5,10,30$ and 50 dpi).

Strain KO348 presented higher densities in the rhizoplane than in the root endosphere at the four time points analyzed
(Figs. 4 and 5). At 5 and $10 \mathrm{dpi}$, it was mainly found at the root hair zone, secondary root mergence, and on grain surface, presenting clear bacterial aggregation, especially at the root hair zone, at $10 \mathrm{dpi}$ (Fig. 4). At $30 \mathrm{dpi}$, we also observed high densities of bacterial aggregation on the rhizoplane that was less evident at 50 dpi (Fig. 5). Kosakonia sp. KO348 was also

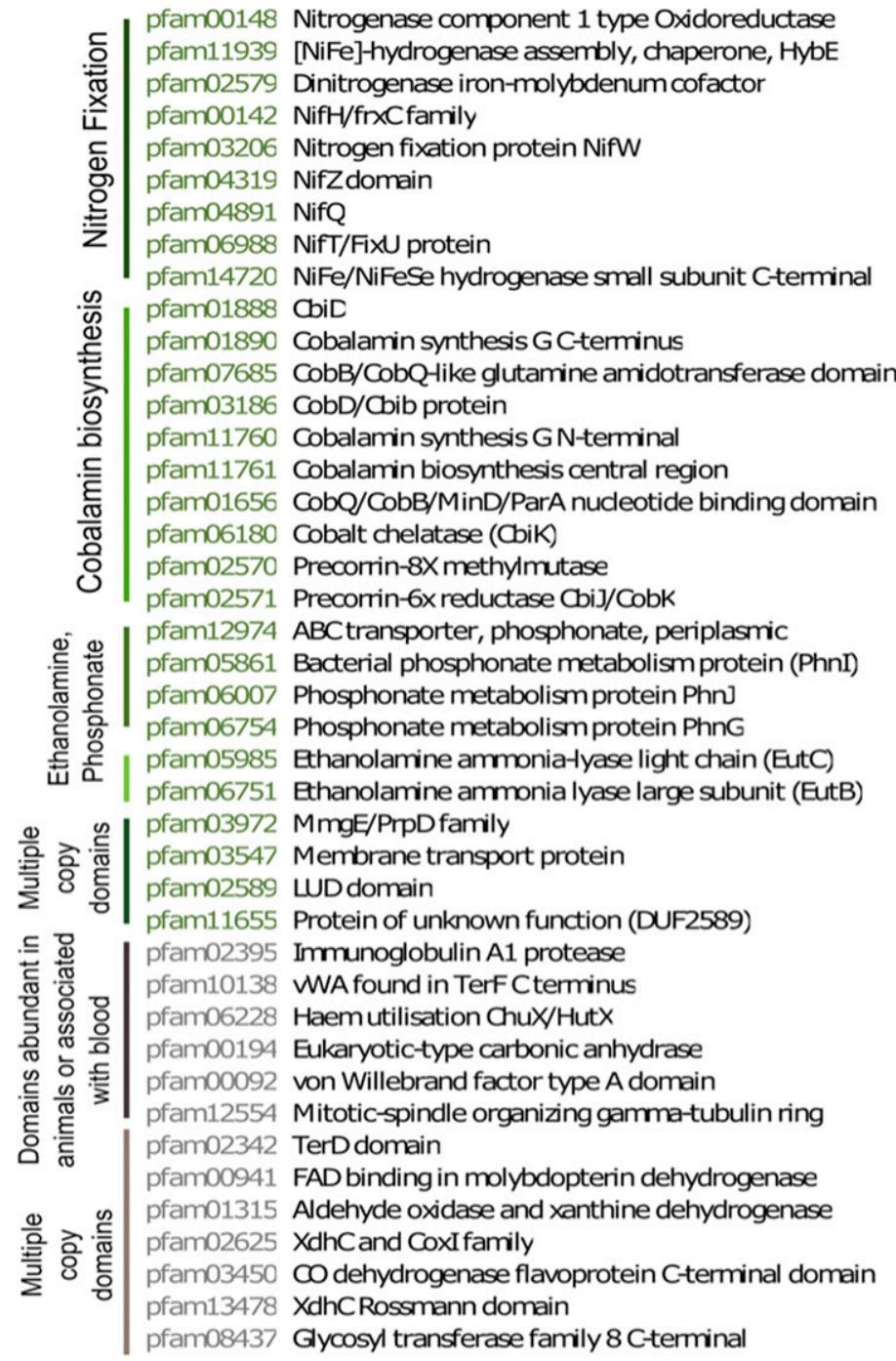

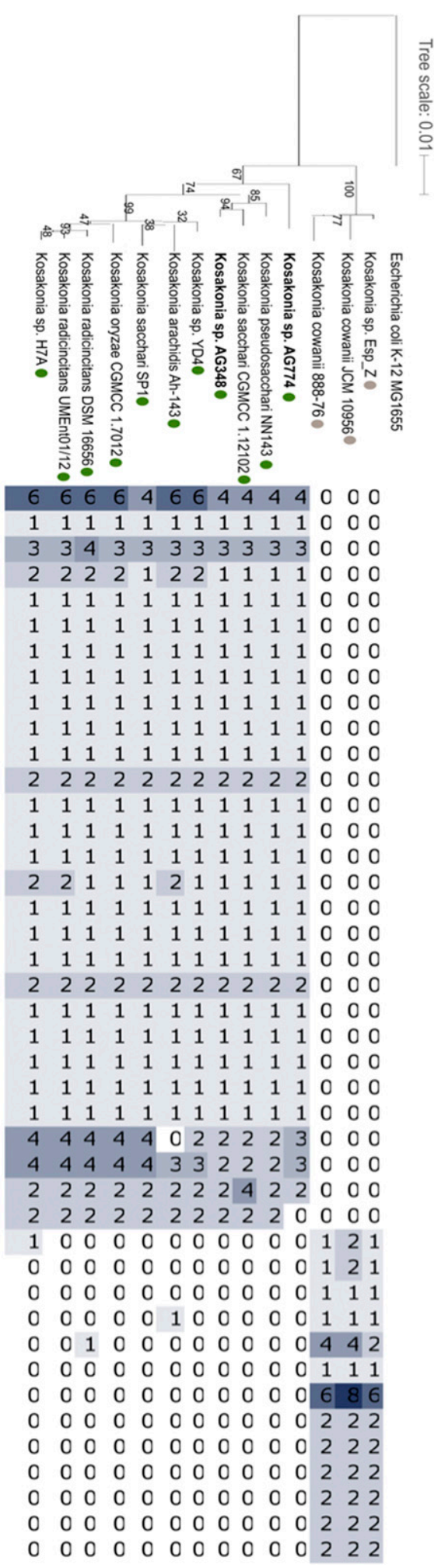

Fig. 1. Kosakonia phylogenetic tree showing enriched protein domains by source of isolation (plants versus human or animals) Phylogenetic tree showing the phylogenetic position of the Kosakonia sp. strains KO348 and KO774 based on 23 single-copy genes found among the 14 complete Kosakonia genomes from the Integrate Microbial Genomes dataset. Escherichia coli K-12 MG1655 was used as an outgroup. Enriched protein domains in the plant-associated Kosakonia versus human- or animal-isolated Kosakonia strains are shown by genome. KO774 is indicated as AG774 and KO348 as AG348. 
observed as endophyte in the transversal root sections, up to the arenchyma, at all the four time points, determining that the strain was able to colonize the root endosphere (Fig. 4 and 5). The uninoculated plants did not present fluorescence at any time point analyzed in either the rhizoplane or the root endosphere (Fig. 5; Supplementary Fig. S2). Therefore, it was concluded that $\mathrm{KO} 348$ was able to attach and colonize the rhizoplane of rice plants and as endophyte forming communities observable until $50 \mathrm{dpi}$.

\section{Secretome profile determination of Kosakonia KO348.}

It was of interest to determine which proteins Kosakonia sp. KO348 produced and secreted in the extracellular medium because these could play a role in the endophytic colonization process. In total, 144 putative secreted proteins were detected

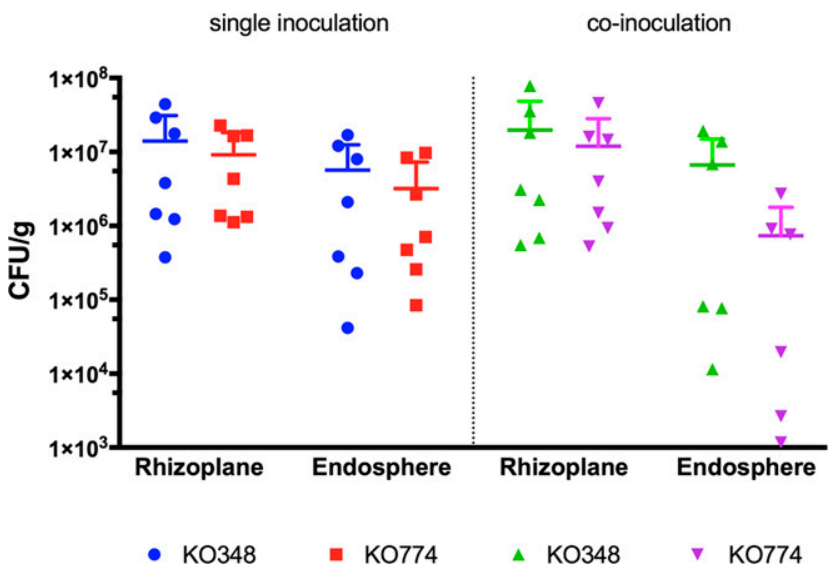

Fig. 2. Rhizoplane and root endosphere colonization by the two Kosakonia strains. Rhizoplane and root endosphere colonization by Kosakonia strains was evaluated in rice plants at 14 days postinoculation by antibiotic selection (KO348 rifampicin resistant and KO774 streptomycin resistant). Three treatment groups were evaluated: KO348 and KO774 in single inoculation $\left(1 \times 10^{8} \mathrm{CFU} / \mathrm{ml}\right)$ and a third group of plants coinoculated with each strain at $0.5 \times 10^{8} \mathrm{CFU} / \mathrm{ml}$. Three biological replicates were performed at different times. Each handled sample consisted of roots of four rice plants. The first biological replicate was performed with 12 plants per group (three handled samples) while the second and the third were performed with 8 plants (two handled samples each). A Kruskall-Wallis test was performed between strains in single and coinoculation; no significant differences were found.

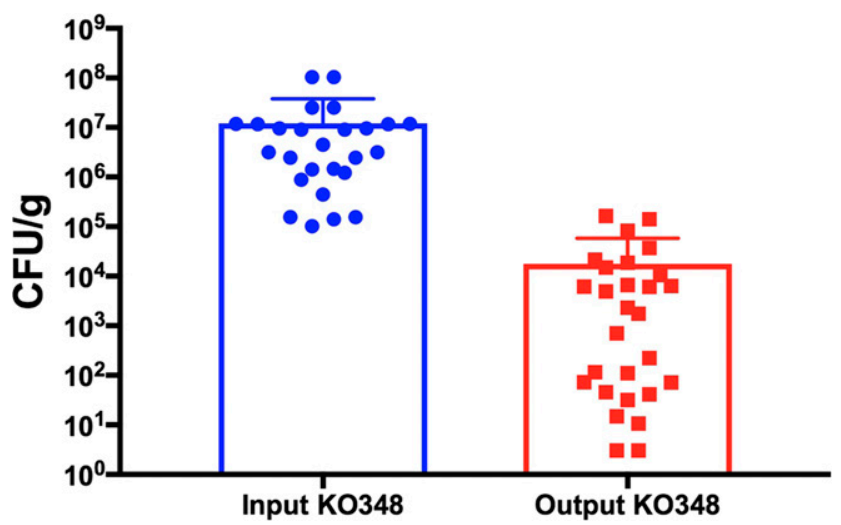

Fig. 3. Endophytic colonization by Kosakonia strain KO348. The endosphere colonization of strain $\mathrm{KO} 348$ was evaluated in roots of rice plants at 30 days postinoculation by plating serial dilutions from previously sterilized plant tissues. The endosphere colonization was evaluated in roots of three different biological replicates performed at different times. Each replicate consisted of 10 plants and all plants were analyzed for CFU per gram independently for confirming colonization ability. when strain KO348 was grown in a minimal plant-mimicking medium (Table 1; Supplementary Tables S3 and S4). Among the ones found were 10 flagella-related proteins (FliD, FliK, FlgK, FlgL, FlgE, FlgJ, FlhA and 3 different proteins of flagellin) and 5 proteins belonging to the type VI secretory system (Hcp, a protein with an FHA domain, and three different proteins of VgrG) (Table 1).

\section{Role of type VI secretory system in rhizoplane and root endosphere colonization in Kosakonia sp. KO348.}

The secretome profile of strain KO348 determined in this study demonstrated that several proteins of the type VI secretion system (T6SS) were present (discussed above). A database search for T6SS domains among the Kosakonia available genomes at the IMG/M showed that the T6SS was present among all genomes, including the two strains of this study (Supplementary Table S5). We searched for T6SS known annotations, found two loci and, in one of them, identified a complete gene cluster of T6SS of strain KO348 (Supplementary Fig. S3). We further searched for putative T6SS effectors by aligning the 70 proteins found in the T6SS loci (Supplementary Table S6) against the proteins identified in the secretome and it was then shown that three proteins matched; two were T6SS VrgG tip proteins (gi|780193605 and gi|780193664) and one the secretion system-associated FHA domain protein TagH (gi|780193691).

Because T6SS component proteins and candidate effectors were expressed in plant-mimicking medium, it was of interest to determine the possible role of T6SS in rhizoplane and root endosphere colonization. A knock-out mutant of the T6SS hcp gene (responsible for the formation of the needle-like structure for the passage of the effectors) called KO348hcp and its complement KO348hcp(pBBRhcp) (carrying a plasmid with the complete $h c p$ gene) were generated. Growth curves of the three strains-KO348, KO348hcp, and KO348hcp(pBBRhcp)-were performed in triplicate in Luria-Bertani (LB) media; the three strains had comparable growing rates or curves (Supplementary Fig. S4). In order to assess the rice colonization ability of the hcp knock-out mutant of Kosakonia sp. KO348, the four following inoculation groups were performed; the KO348 wild-type (WT), the KO348hcp mutant, the complemented hcp mutant KO348hcp(pBBRhcp), and the KO348 WT and mutant KO348hcp together in a competition experiment. For all four groups, the same amount of total bacteria $\left(1.3 \times 10^{7} \mathrm{CFU} / \mathrm{ml}\right)$ was used for plant inoculation. In the case of the competition experiment, we used each strain at $0.65 \times 10^{7} \mathrm{CFU} / \mathrm{ml}$.

In planta experiments determined that, at $14 \mathrm{dpi}$, the colonization of the rhizoplane by WT strain KO348 was nearly 70fold higher than the colonization of the hcp mutant KO348hcp $\left(2.3 \times 10^{6}\right.$ versus $3.3 \times 10^{4} \mathrm{CFU} / \mathrm{g}$ of root, respectively) (Fig. 6). A significant difference was also observed between the WT KO348 and the hcp mutant when they where coinoculated $\left(7.2 \times 10^{5}\right.$ versus $3.6 \times 10^{4} \mathrm{CFU} / \mathrm{g}$ of root, respectively). Complementing the mutant with the $h c p$ gene harbored in a plasmid resulted in restoration of its ability to colonize the rhizoplane $\left(2.3 \times 10^{5} \mathrm{CFU} / \mathrm{g}\right)$ (Fig. 6A). In this complementation experiment, the percentage of bacterial strains that retained the plasmid was $78 \%$, indicating a low incidence of plasmid loss. Rhizoplane colonization was not affected by the presence of the empty plasmid vector in the mutant strain KO348hcp(pBBRMCS-1); the plasmid retention was $69 \%$ (Supplementary Fig. S5).

We also performed studies of root endosphere colonization and results showed a significant difference between the colonization ability of the WT and the $h c p$ mutant in plants inoculated independently $\left(6.4 \times 10^{4}\right.$ versus $2.2 \times 10^{3} \mathrm{CFU} / \mathrm{g}$ of root, respectively). This significant difference between the WT and mutant was maintained when plants were coinoculated 
$\left(2.7 \times 10^{4}\right.$ versus $1.4 \times 10^{4} \mathrm{CFU} / g$ of root, respectively) (Fig. $6 \mathrm{~B})$. However, unlike in the rhizoplane experiment, the complemented mutant did not result in the restoration of endophytic colonization to WT levels $\left(4.9 \times 10^{2} \mathrm{CFU} / \mathrm{g}\right)$. The low plasmid retention $(22 \%)$ at the root endosphere colonization level was also observed in the mutant strain harboring the empty plasmid vector KO348hcp(pBBRMCS-1). The hcp mutant strain not carrying the plasmid was significantly more recovered in the root endosphere than the mutant harboring the empty vector KO348hcp(pBBRMCS-1), indicating plasmid loss (Supplementary Fig. S5). In this case, the percentage of bacterial cells which retained the plasmid harboring the hcp gene was only $23 \%$; this is the most likely the reason for the lack of complementation. In summary, these results suggest a significant

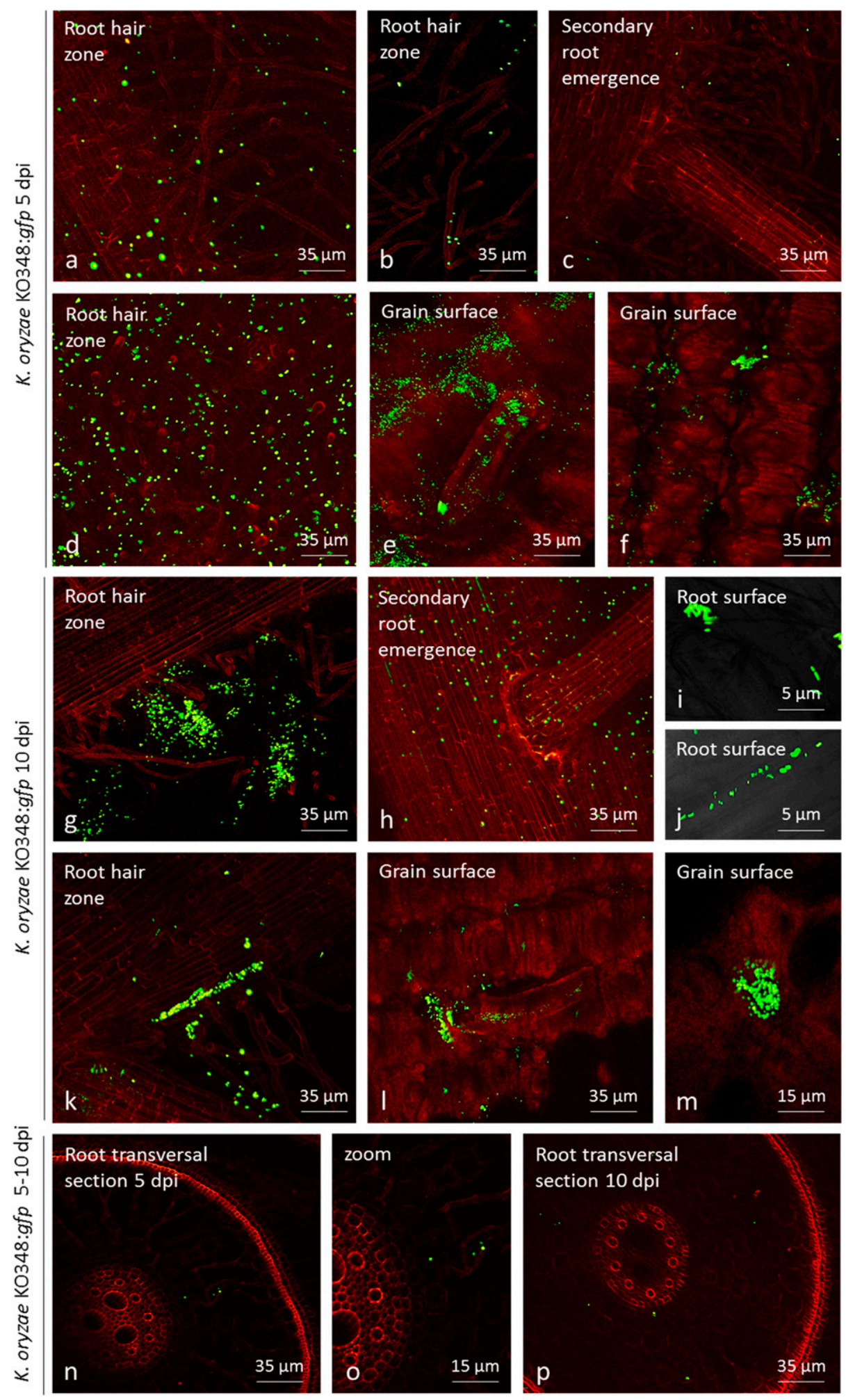

Fig. 4. Microscopic visualization of Kosakonia strain KO348(pBBRgfp) in rice roots at rhizoplane and root endosphere level at 5 and 10 days postinoculation (dpi). KO348(pBBRgfp) was visualized by fluorescence microscopy at 5 and $10 \mathrm{dpi}$ in the root hair zone (rhizoplane) and in the root aerenchyma (endosphere). In total, 10 plants were analyzed for each time point. 
role for T6SS in the rhizoplane colonization and, to a much lesser extent, in the root endosphere colonization by Kosakonia sp. KO348.

Field rice inoculation with Kosakonia sp. KO774.

Because it was previously determined that the diazotrophic Kosakonia strains studied here displayed plant-growth- promoting properties (Bertani et al. 2016), it was of interest to perform a field rice experiment in order to assess whether it could compensate for a reduction in nitrogen fertilization. Between May and October 2016 in Valencia, Spain, we performed a rice field trial with diazotrophic Kosakonia sp. KO774 with the aim of testing whether rice seed inoculated with the bacterial strain can compensate for a $50 \%$ reduction
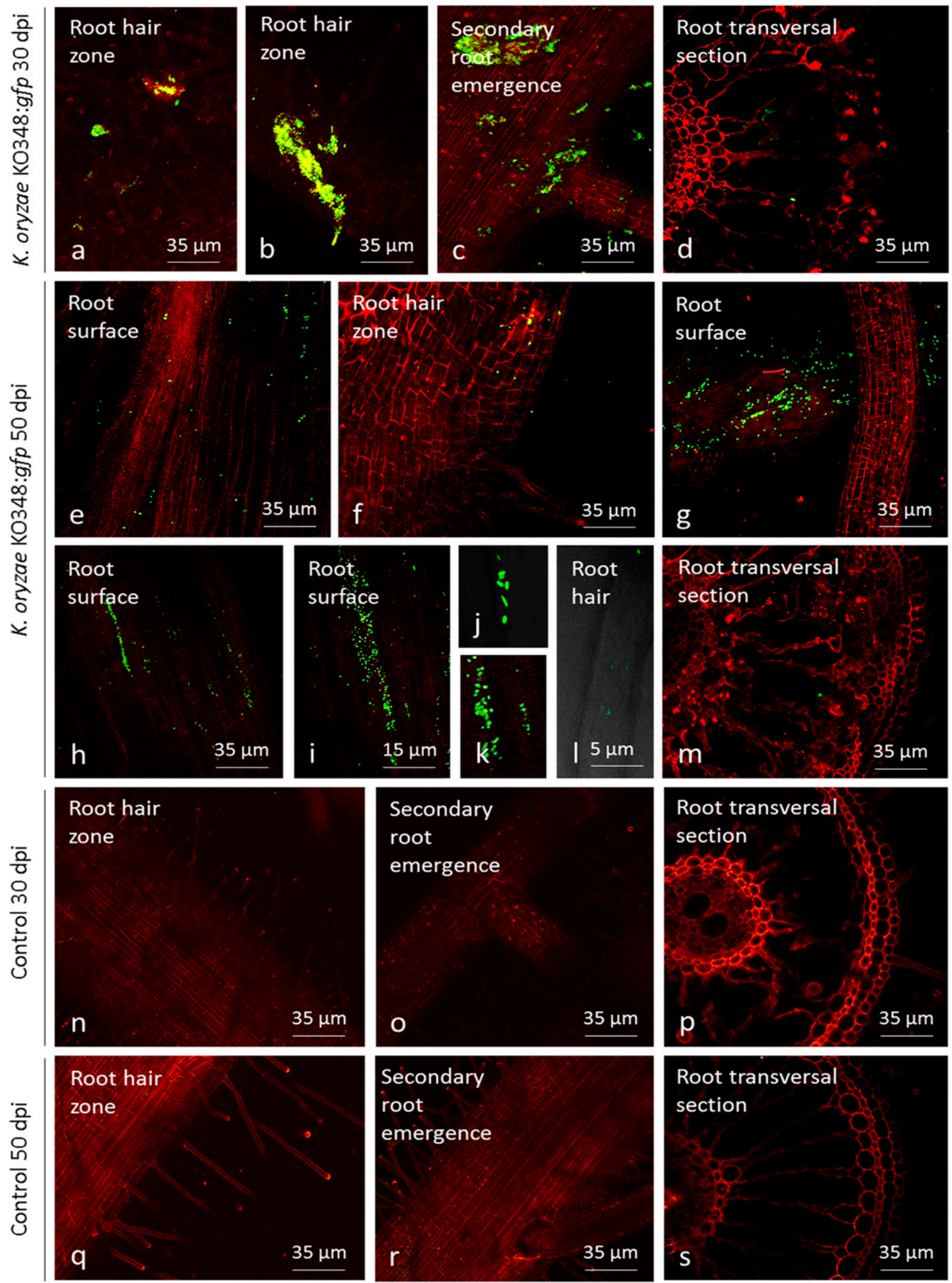

Fig. 5. Microscopic visualization of Kosakonia strain KO348(pBBRgfp) in rice roots at rhizoplane and root endosphere level at 30 and 50 days postinoculation (dpi). KO348(pBBRgfp) was visualized by fluorescence microscopy at 30 and 50 dpi in the root hair zone (rhizoplane) and in the root aerenchyma (endosphere) and compared with control plants. In total, 10 plants were analyzed for each time point. 
in nitrogen fertilization. In total, 16 growth plots were grown, which were divided into four groups: (i) 8 plots receiving $100 \%$ nitrogen/urea fertilization, 4 planted with seed inoculated with strain $\mathrm{KO} 774$, and the other 4 with the seed not inoculated and (ii) 8 plots receiving only $50 \%$ of urea/nitrogen fertilization, 4 of these planted with seed inoculated with strain KO774 and the other 4 with the seed not inoculated (Supplementary Fig. S6).
All of the plots were harvested 100 days postsowing and different growth parameters such as germination/plot, weight of 1,000 grains/plot and 25 panicles/plot, and yield (kilograms per hectare) were then assessed or measured (Table 2). No statistically significant differences in any of the measured parameters were found between the inoculated and uninoculated plots. This indicated that inoculation with Kosakonia spp. did not result in any plant growth promotion or

Table 1. Flagella-related proteins and type VI secretion system (T6SS)-related proteins in the Kosakonia KO348 secretome

\begin{tabular}{|c|c|c|c|}
\hline Uniprot ID & Protein name & Unique peptides & $\log (e)^{a}$ \\
\hline \multicolumn{4}{|c|}{ Flagella-related proteins } \\
\hline A0A369A2B9 & Flagellin & 73 & -820.4 \\
\hline A0A368ZZS7 & Flagellar hook-associated protein 2 & 64 & -743.7 \\
\hline A0A369ACC2 & Flagellar hook-associated protein $1(\mathrm{FlgK})$ & 47 & -556.8 \\
\hline A0A368ZXF8 & Flagellin-like protein (Fragment) & 4 & -471.4 \\
\hline A0A369ADA8 & Flagellin & 5 & -261 \\
\hline A0A368ZX89 & Flagellar hook-length control protein FliK & 15 & -113.9 \\
\hline A0A369A9W2 & Flagellar hook-associated protein $3 \mathrm{FlgL}$ & 11 & -100.6 \\
\hline A0A369A9A4 & Flagellar hook protein FlgE & 2 & -11.6 \\
\hline A0A368ZXA5 & Flagellar biosynthesis protein FlhA & 3 & -11.5 \\
\hline A0A369ACY1 & Flagellar protein FlgJ & 2 & -6.8 \\
\hline \multicolumn{4}{|l|}{ T6SS proteins } \\
\hline A0A368ZWR4 & T6SS-secreted protein Hcp & 7 & -61.9 \\
\hline A0A368ZZA3 & T6SS-secreted protein VgrG & 8 & -47 \\
\hline A0A368ZRA6 & T6SS-secreted protein VgrG & 3 & -42.1 \\
\hline A0A368ZYZ5 & Rhs element Vgr protein (fragment) & 2 & -28.3 \\
\hline A0A368ZZB3 & FHA domain protein & 3 & -11.1 \\
\hline
\end{tabular}

${ }^{a}$ Statistical confidence.
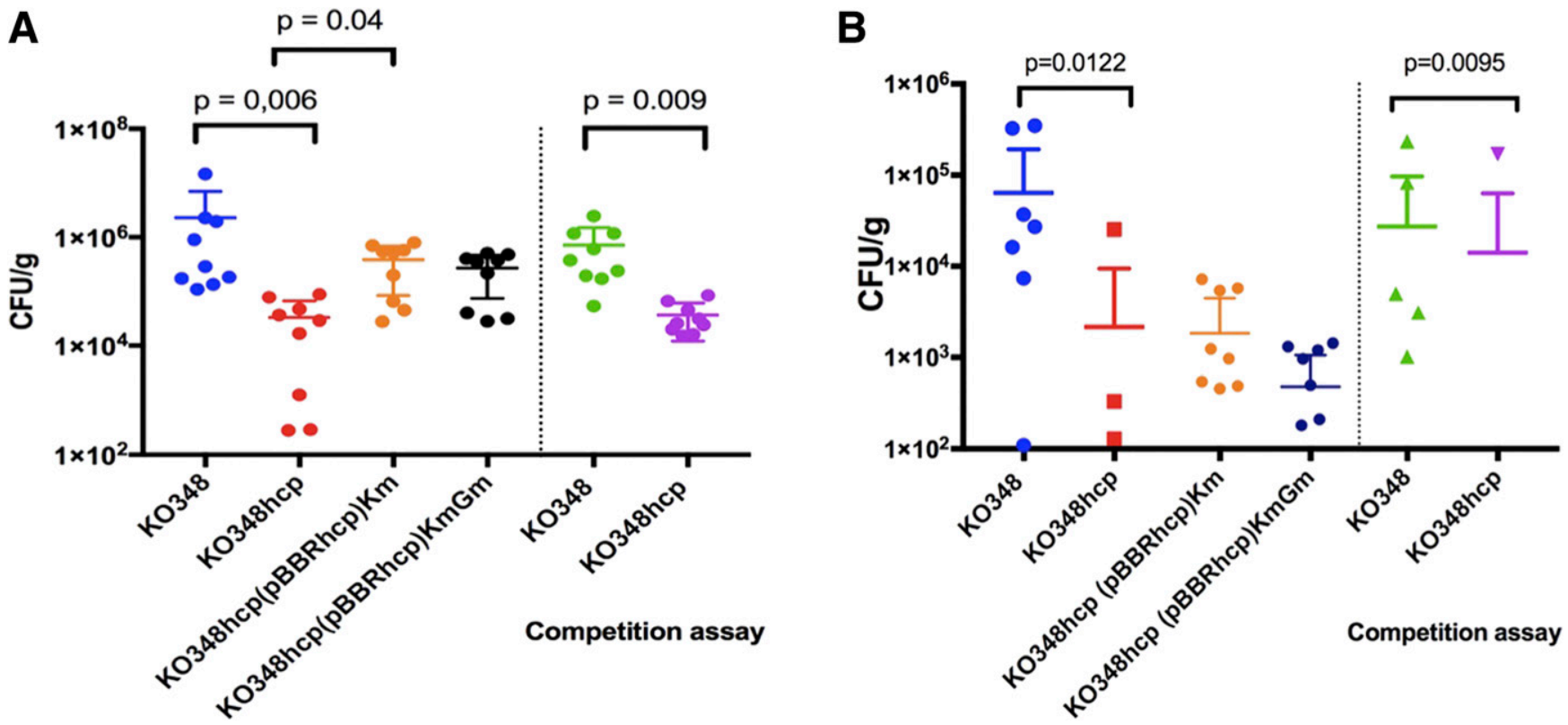

Fig. 6. Role of the type VI secretion system (T6SS) of Kosakonia KO348 in rhizoplane and endosphere rice root colonization. The effect of T6SS was tested in $\mathbf{A}$, the rhizoplane and $\mathbf{B}$, the endosphere colonization of rice root plants at 10 days postinoculation. The KO348hcp mutant was inoculated independently and in competition assays (in the same rice plant) with the KO348 wild type. Three different biological replicates were performed at different times. In A, each replicate had three plants analyzed individually; in B, each replicate had four plants analyzed individually. For the calculation of plasmid loss during rhizoplane and root endosphere colonization, the complemented mutant KO348hcp(pBBRhcp) was plated in kanamycin (Km) for KO348hcp and in Km and gentamicin (Gm) for KO348hcp(pBBRhcp).

Table 2. Field trial biological parameters by group

\begin{tabular}{lcccc}
\hline & Weight (1,000 grains/plot) & Weight $(\mathbf{2 5}$ panicles/plot) & Yield $\left(\mathbf{m}^{2}\right)$ & Yield $(\mathbf{k g} / \mathbf{h a})$ \\
\hline Nitrogen 100\% & 35 & 96.8 & 958.8 & $9,587.5$ \\
Nitrogen 100\%+ KO774 & 35 & 90 & 943.8 & $9,437.5$ \\
Nitrogen 50\% & 34.3 & 98.8 & 853.5 & 8,585 \\
Nitrogen 50\% + KO774 & 35.3 & 101.3 & 828.5 & 8,285 \\
\hline
\end{tabular}


nitrogen biofertilization under the conditions tested (Table 2).

\section{Rice microbiome analysis of the rice field trial using} seed inoculated with Kosakonia sp. KO774.

Following the rice seed inoculation with Kosakonia sp. KO774 in the field trial (discussed above), it was also of interest to determine the colonization of strain KO774 and the effect on the total endospheric microbial community of rice plants. We determined the root endomicrobiome at three different time points $(30,60$, and 90 days postsowing) in plants which had been fertilized with $50 \%$ of the recommended amount of nitrogen and had been inoculated with $\mathrm{KO} 774$ as well as in ones that were not.
In the endomicrobiome, we first determined the presence of bacterial sequences with $100 \%$ identity to the $16 \mathrm{~S}$ ribosomal DNA gene fragment of Kosakonia sp. KO774. This sequence was present in all three samples in the group with Kosakoniainoculated seed at $30 \mathrm{dpi}$; however, at $60 \mathrm{dpi}$, we only observed the sequence at much lower levels in one of the three samples. At 90 dpi, this $16 \mathrm{~S}$ ribosomal RNA (rRNA) gene sequence of Kosakonia sp. KO774 was not detected (Supplementary Fig. S7). In the group which was not seed inoculated with strain KO774, the $100 \%$ identity Kosakonia DNA sequence was observed in only one sample at $90 \mathrm{dpi}$, and at low abundance (Supplementary Fig. S7).

A total root endomicrobiome analysis was also performed to analyze the main bacterial genera ( $>1 \%$ abundance) at $30 \mathrm{dpi}$,
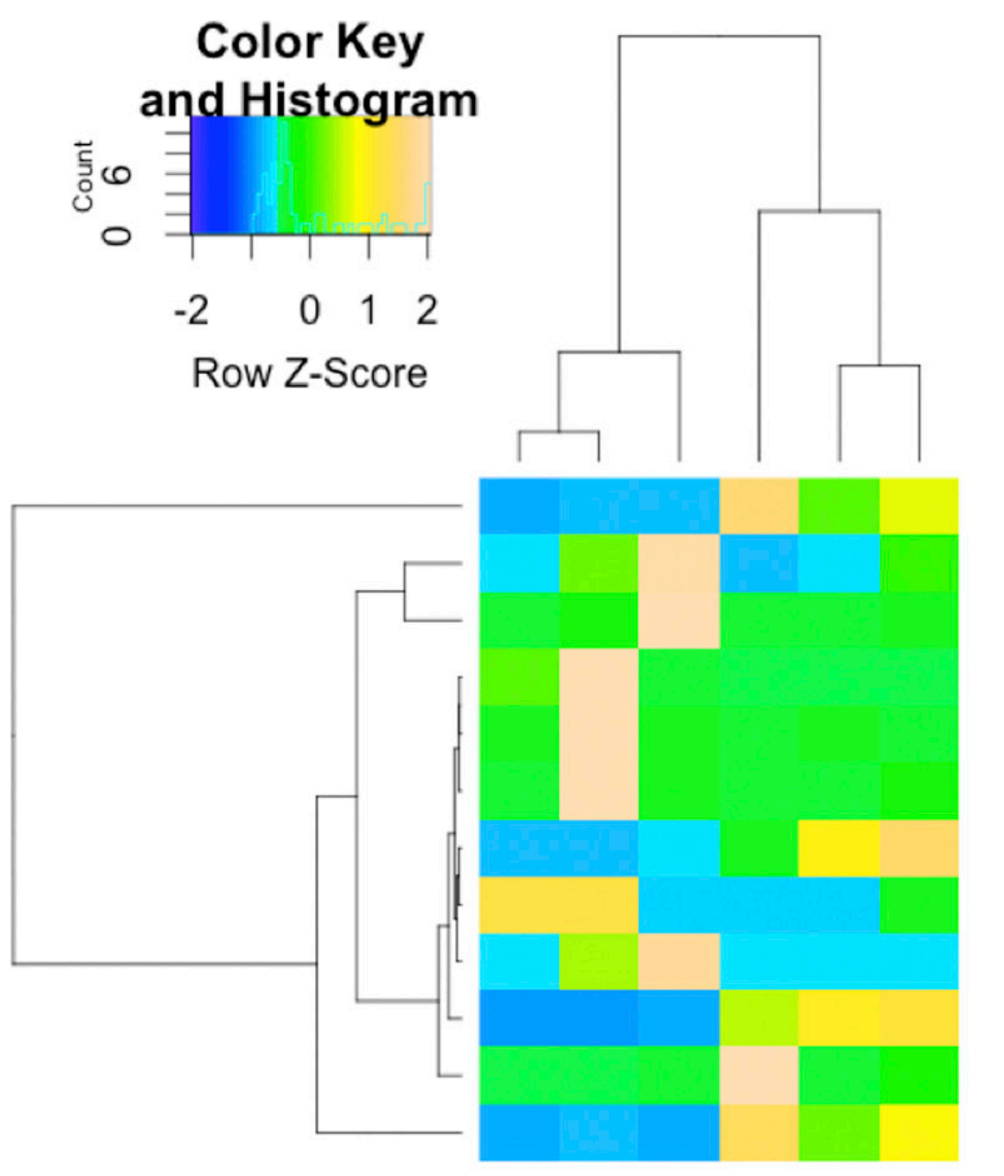

Enterobacteriales Enterobacteriaceae NA

Enterobacteriales Enterobacteriaceae Pantoea

Bacteroidales Marinilabiliaceae NA

Actinomycetales Thermomonosporaceae Actinocorallia

Desulfobacterales Desulfobacteraceae Desulfobacula

Desulfobacterales Desulfobacteraceae Desulfatitalea

Caulobacterales Caulobacteraceae Asticcacaulis

Burkholderiales Burkholderiales_incertae_sedis Piscinibacter

Desulfobacterales Desulfobulbaceae NA

Rhodospirillales Rhodospirillaceae Rhodospirillum

Family_XIII GpXIII NA

Enterobacteriales Enterobacteriaceae Kosakonia

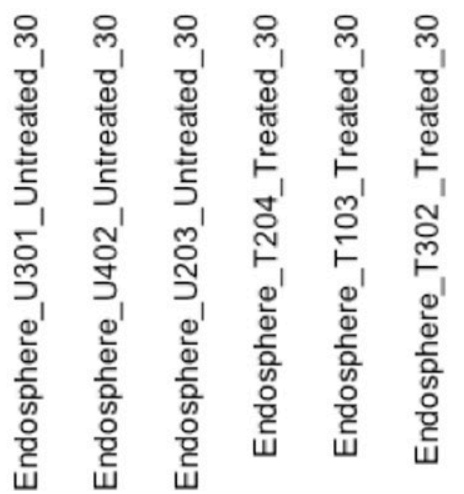

Fig. 7. Heatmap of the most abundant genus $(>1 \%)$ by sample and treatment at 30 days postinoculation in the field trial. The clustering of the most abundant bacterial genera and operational taxonomic units present in rice root endosphere at 30 days postinoculation among different samples is shown in the heatmap. The heat map scale displays the row $\mathrm{Z}$ score, where the $\mathrm{Z}$ score is calculated as (relative abundances of a genus in one sample - mean relative abundance of the same genus among total samples)/standard deviation. 
when Kosakonia sp. KO774 was still present. It was observed that the hierarchical clustering positioned the samples closer within each treatment group, indicating that all seed-inoculated samples were clustered together and all the uninoculated samples were closer among them (Fig. 7). However, when observing the $\mathrm{Z}$-scores based on distribution and relative abundance of each genus, we observed a significant difference between the two group sets (inoculated versus uninoculated) in only a few genera such as Kosakonia (as expected), Rhodospirillum, Asticcacaulis, and Enterobacteriaceae NA (Fig. 7).

When analyzing the clustering and patterns of distribution of all samples by treatment and time point by nonmultidimensional scaling analysis, it was observed that all samples were mainly clustered by time point, with seed inoculation of Kosakonia sp. KO774 not being a major factor for clustering. One sample at $30 \mathrm{dpi}$ belonging to the untreated group can be clearly identified as an outlier (Fig. 8).

These findings suggested that Kosakonia sp. KO774 was able to colonize the rice root endosphere at the given conditions only at the first time point analyzed (30 dpi) after seed sowing and that the endosphere microbial community was not significantly affected by the inoculation.

\section{DISCUSSION}

Different strains of the recently described genus Kosakonia have been isolated from crops and vegetables (Berger et al. 2018; Bergottini et al. 2015; Kämpfer et al. 2016; Shinjo et al.
2016; Witzel et al. 2012) and many possess plant-beneficial phenotypes such as nitrogen fixation and phosphate solubilization. In this study, we report the characterization of two endophytic diazotrophic Kosakonia strains.

Genome analysis of some members of the Kosakonia genus, including strains KO774 and KO348 studied here, has revealed that they share some common genetic loci such as enzymes which can facilitate endophytic colonization by degrading plant-cell-wall polysaccharides or by removing reactive oxygen species (Li et al. 2017; Reinhold-Hurek and Hurek 2011). In the case of $\mathrm{KO} 774$, its genomic analysis revealed a plasmid containing a putative large cellobiose gene, an enzyme possibly involved in cellulose degradation; a similar enzyme has been previously reported in other endophyte, Enterobacter sp. 638 (Taghavi et al. 2015). A recent comparative genomics study based on $K$. radincincitans DSM 16656 described multiple flagellar and secretion systems contributing to high motility and high competitiveness, thus increasing bacterial fitness (Becker et al. 1997). Comparative genomics revealed that enriched protein domains include the nitrogen fixing cluster (nif regulon), which has been reported in diverse Kosakonia plant-associated strains (Becker et al. 1997). Other enriched protein domains include functions related to cobalamin biosynthesis; this is an enzyme cofactor synthesized only by prokaryotes which in Sinorhizobium meliloti involved in symbiosis and nodule formation (Taga and Walker 2010). Phosphonate metabolism is also enriched in plant-associated Kosakonia strains; phosphonate is a rich source of soil phosphate which

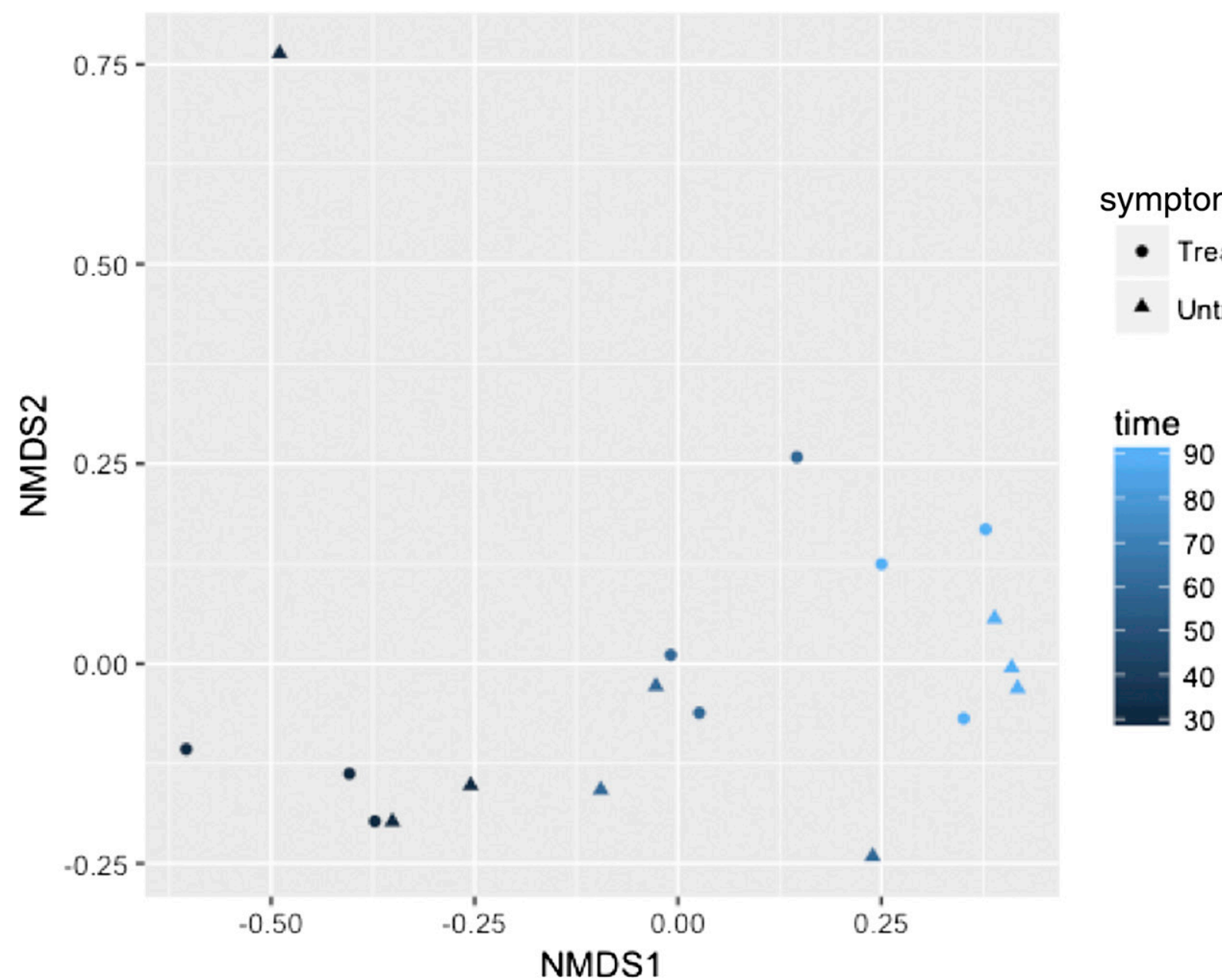

Fig. 8. Distribution patterns analysis by nonmultidimensional scaling (NMDS) analysis of the microbiomes by time and treatment. NMDS analysis plot showing clustering of samples of rice endophytic microbial communities by time and treatment based on Bray-Curtis dissimilarity. 
plays a role in plant-bacteria interactions (Kamat and Raushel 2013). Phosphonate utilization strains of Stenotrophomonas rhizophila, Cupriavidus basilensis, and Caulobacter segnis, among others, have been isolated from the rhizoplane of Lolium perenne (Fox et al. 2014). Finally, some ethanolamine utilization protein domains were also enriched; ethanolamine can be used in some bacteria as a valuable source of carbon and nitrogen (Kaval and Garsin 2018). Ethanolamine utilization is important for bacterial pathogens of animals and plants (for example, in the plant pathogen Erwinia chrysanthemi) (Kaval and Garsin 2018). Interestingly, recently, ethanolamine and derivatives have been linked to plant bacterial interkingdom signaling (Coutinho et al. 2018).

Previous studies have shown that good endosphere colonizers are recovered in vitro on the order of $10^{4}$ to $10^{6} \mathrm{CFU} / \mathrm{g}$ after more than 1 week postinoculation (Luna et al. 2010; Schmidt et al. 2011). Colonization studies performed here indicate that the two Kosakonia strains are very efficient root endosphere and rhizoplane colonizers. Fluorescence microscopy visualization also confirmed the KO348 ability to colonize the rice roots endosphere. $K$. radicincitans DSM 16656 has also been recently observed in the root cortex of cucumber by confocal microscopy (Sun et al. 2018). The weak green fluorescent protein (GFP) signal observed in the endosphere could be due to plasmid loss, as we have also observed in this study. Other studies have shown that transmission electron microscopy allowed the localization of bacterial diazotrophs in rice in the apoplasm (Egener et al. 1999; Gyaneshwar et al. 2001; Hurek et al. 1994).

Endophytes are likely to have evolved an intimate relationship with their plant host, probably involving interkingdom signaling (Lòpez-Fernàndez et al. 2017; Reinhold-Hurek and Hurek 2011). We detected approximately 144 putative secreted proteins of Kosakonia sp. KO438 and many of these can be involved in plant-bacteria interactions; flagellar and T6SS proteins were among the most abundant found. The secretome profile contained several membrane-associated proteins; these are not necessarily secreted because some proteins can end up in the supernatant because they are loosely associated with the membrane or due to cell lysis. A similar secretome analysis has been performed in the endophyte $H$. seropedicae SmR1, and 41 secreted proteins have been reported, including 19 flagellarelated proteins (Chaves et al. 2009). No proteins belonging to the T6SS have been found in the secretome of $H$. seropedicae; however, the presence of T6SS in genomes is very common among plant-associated bacteria (Levy et al. 2018) and in endophytes (Frank 2011). Interestingly, T6SS genes have been found enriched in the rhizoplane of barley (Bulgarelli et al. 2015). The T6SS is a phage-like secretion system found in approximately $25 \%$ of Gram-negative bacteria, mainly in Proteobacteria, including many plant-associated bacteria (Bernal et al. 2018; Boyer et al. 2009). Interestingly, it is a hostspecificity factor in the symbiont Rhizobium leguminosarum (Van Brussel et al. 1986). K. radicincitans possesses three different types of T6SS; however, their function or mechanism of action have not been studied (Becker et al. 1997). T6SS in endophytes can be involved in host interaction or antagonizing other microbes in the endosphere (Frank 2011). Here, we report that a T6SS mutant of Kosakonia sp. KO348 displayed a significant decrease in rice rhizoplane and root endosphere colonization, thus suggesting a role in the host-bacteria colonization or interaction. The hcp mutant was not affected in bacterial growth under the conditions tested; hence, the T6SS system does not play a role in bacterial growth. A limitation in the T6SS root endosphere colonization experiment was that the many Kosakonia cells in planta lose the pBBR1MCS-5 plasmid vector, regardless of whether or not it harbors the hcp gene.
This vector is stable in Kosakonia KO348 in laboratory media; thus, it is lost during Kosakonia in planta colonization due to the lack of selection and, therefore, affecting the studies involving complementation of genomic mutants.

Nitrogen is one of the most used fertilizers worldwide for all cereals, including rice, with an annual growing rate of utilization of 1.9, and it is expected that 201.66 million tons will be used in 2020 (FAO 2017). Biofertilizers are considered an alternative to decrease the use of chemicals; however, only a few reports of greenhouse and field trials using rhizospheric or endospheric diazotrophic strains have been performed thus far. Most of these are in wheat or maize and report an increase between 6 and 33\% in total yield (Santi et al. 2013). Furthermore, plant-associated microorganisms applied in agriculture as biofertilizers or biopesticides are usually subject to a rigorous risk assessment which requires a better understanding of the mechanisms involved in the mutualism to facilitate and promote the development and application of sustainable microbial solutions in crop production (Brader et al. 2017). The associative microbial nitrogen fixation supplied by microbes in rice is predicted to be between 20 and $25 \%$ of the total nitrogen needed by the plant (Ladha et al. 1987; Saikia and Jain 2007). Inoculation experiments using $H$. seropedicae or Burkholderia spp. revealed that 11 to $20 \%$ of the total nitrogen accumulated in rice plants can be attributed to the bacterial strains (Divan Baldani et al. 2000). Similarly, inoculation studies using $K$. radicincitans (DSM 16656) increased plant root or shoot dry weight by $150 \%$ under high nitrogen conditions (350 mg/plant) and $130 \%$ in low nitrogen conditions (150 mg/plant) (Berger et al. 2013). This latter study also showed that plants with low nitrogen supplementation increased the pathogen defenserelated markers and suggested that this plant response could negatively affect or inhibit the PGP effect of Kosakonia. A recent report using the AbiVital product $(67 \% \mathrm{~K}$. radicincitans DSM $16656^{\mathrm{T}}$ and $37 \%$ cryopreservation additives) in maize resulted in an increase in yield of approximately $30 \%$ in field trials, including organic and conventional cultivation systems (Berger et al. 2018). In our field trial, we decreased the nitrogen fertilization by $50 \%$, hoping that supplementation via seed inoculation of the Kosakonia strain could, at least in part, overcome nitrogen deficiency; this was not the case in any of the measurements performed. This experiment could have benefited from knowing the nitrogen concentration in the soil used for the field trial. We observed that, under the conditions used, the colonization of the inoculated strain was rather inefficient over a longer period of time (more than 30 days) and that it did not affect the root endosphere microbiome; thus, a possible limiting factor was likely to be the establishment of the strain in the plant endosphere. This could have been due to inoculation method, soil microbial community, or abiotic factors which are not favorable for the Kosakonia strain that we used. Interestingly, Becker et al. (1997) reported a significant impact on the bacterial community composition of tomato following inoculation of $K$. radicincitans DSM $16656^{\mathrm{T}}$. This field trial was performed in Spain on a different rice variety used in the laboratory experiment; hence, colonization efficiencies of Kosakonia spp. could be different from the ones reported in this study. However, other members of genus Kosakonia have been shown not to display host specificity and, in general, endophytes are generalists, being able to colonize many different hosts (Compant et al. 2005; Ma et al. 2011).

Due to the recent description of the genus Kosakonia (Brady et al. 2013), there are only a few reports on the presence of Kosakonia spp. in the rice microbiome. Kosakonia spp. are dominant colonizers in seed of three salt-tolerant rice varieties (Walitang et al. 2017, 2018). In addition, when inbreeding seed varieties containing Kosakonia spp., they were then present 
with a similar abundance or at even higher levels within the offspring, suggesting that genus Kosakonia is part of the core microbiota of some rice varieties (Walitang et al. 2019). Kosakonia spp. have been isolated from different rice varieties, largely representing approximately one-third of the total isolates (Hardoim 2015). This indicates that members of genus Kosakonia are common endophytes of rice.

This work has characterized two Kosakonia strains, providing some highlights of their interaction with the plant host and its colonization. Further studies on the genus Kosakonia are important for understanding the mechanisms that allow members of this genus to be successful endophytic colonizers and be part of the microbiome of economically important crops.

\section{MATERIALS AND METHODS}

\section{Bacterial strains and growth conditions.}

The Kosakonia strains used in this study (KO348 and KO774) were previously isolated from the root endosphere from rice grown in Italy (Bertani et al. 2016). Strains KO774, KO348, KO348(pBBRgfp), KO348hcp, and KO348hcp(pBBRhcp) were routinely grown in LB broth at $30^{\circ} \mathrm{C}$. In order to obtain spontaneous rifampicin (Rif)-resistant $\mathrm{KO} 348$ and streptomycin (Sm)-resistant KO774, strains were grown in one-sixth tryptic soy broth (TSB) medium supplemented with gradually increasing amounts of Rif or Sm, respectively, ranging from 15 to $100 \mu \mathrm{g} \mathrm{ml}^{-1}$. Finally, cultures were plated on tryptic soy agar (TSA) and single colonies were reinoculated in TSB containing Rif at $100 \mu \mathrm{g} \mathrm{ml}^{-1}$ or $\mathrm{Sm}$ at $100 \mu \mathrm{g} \mathrm{ml}^{-1}$. When required, antibiotics for Kosakonia strain growth were added at the following concentrations: Rif at $50 \mu \mathrm{g}$ $\mathrm{ml}^{-1}$, gentamicin $(\mathrm{Gm})$ at $25 \mu \mathrm{g} \mathrm{ml}^{-1}$, and kanamycin $(\mathrm{Km})$ at $100 \mu \mathrm{g} \mathrm{ml}^{-1}$. Escherichia coli DH5 $\alpha$ and $\mathrm{S} 17$ were grown at $37^{\circ} \mathrm{C}$ in LB broth and, when appropriate, antibiotics were added at the following concentrations: ampicillin at $100 \mu \mathrm{g} \mathrm{m}{ }^{-1}$ and $\mathrm{Gm}$ at $15 \mu \mathrm{g} \mathrm{ml}^{-1}$

\section{Genome sequencing of Kosakonia strains.}

The genome of Kosakonia sp. KO348 has been previously sequenced and was deposited at DNA Data Bank of Japan/European Molecular Biology Laboratory/GenBank under the accession number JZLI00000000 (Meng et al. 2015) and in IMG/M (U.S. Department of Energy-Joint Genome Institute) as genome ID 2651869662. We resequenced KO348 using Illumina HiSeq technology at 230x sequencing depth. The genome was assembled using spades v. 3.10.1. The genome was annotated via IMG Annotation Pipeline v.4.16.0. The draft genome sequence of Kosakonia sp. KO774 was also determined in this study. For this, the genomic DNA was obtained by the Sarkosyl-Pronase lysis protocol, as described by Better et al. (1983), then used to prepare a sequencing-ready library. Sequencing was performed on Illumina MiSeq platform using 150-bp paired-end reads. The genome of Kosakonia sp. KO774 was deposited in IMG/M as genome ID 2758568389. Automated annotation of Kosakonia sp. KO774 draft genome sequence was performed using IMG/M (U.S. Department of Energy-Joint Genome Institute).

\section{Kosakonia genome analysis.}

In order to analyze the genomes for a phylogenetic analysis, 15 Kosakonia genome sequences were retrieved from the Integrated Microbial Genomes database IMG/M (U.S. Department of Energy-Joint Genome Institute).

E. coli K12 MG1655 served as an outgroup. The list of single-copy marker genes was retrieved for all genomes and consisted of mainly ribosomal proteins. Only genes that were present in all 15 genomes were used, and these included the following clusters of orthologous groups (COGs): COG0012,
COG0016, COG0052, COG0087, COG0090, COG0091, COG0092, COG0094, COG0096, COG0097, COG0098, COG0099, COG0102, COG0103, COG0124, COG0186, COG0197, COG0200, COG0201, COG0522, COG0525, COG0533, and COG0541. The genes of each COG in all 15 genomes were aligned separately using MAFFT multiple aligner, version 7.221 (K. Katoh and D. M. Standley) using default parameters. The multiple sequence alignment was trimmed with trimAl v1.3 using default parameters. Next, the different COG alignments were concatenated together using a custom script to yield 15 sequences of all 23 singlecopy genes. RAxML version 7.6.3 (The Exelixis Lab, Heidelberg Institute for Theoretical Studies) was used to construct the tree using the following parameters: raxmlHPC-PTHREADS-SSE3-f a-p 12345-x 12345-\# 1000-m PROTGAMMALG-T 8, with the outgroup being $E$. coli. The best-scoring maximum-likelihood tree with support value was visualized using iTOL (I. Letunic and P. Bork).

We retrieved all proteins $(n=70)$ located within and adjacent to the T6SS operons in the KO348 genome and we blasted them with the proteins found in the secretome (discussed below), searching for possible hits which might be T6SS effectors.

\section{Plant colonization experiments.}

For all of the rice endosphere colonization experiments, we followed the inoculation protocol described previously by Bertani et al. (2016), with a few modifications. Kosakonia Rif- or Smresistant strains were grown on LB media to an optical density at $600 \mathrm{~nm}\left(\mathrm{OD}_{600}\right)$ of 0.8 and 7-day-old germinated Baldo rice plantlets were then submerged in this bacterial suspension for $1 \mathrm{~h}$ and transferred independently to a tube containing Hoagland's semisolid solution (Steindler et al. 2009). Plantlets were then watered and grown for a number of days; Kosakonia strains were then reisolated from roots or the green aerial part of the plant after surface sterilization and sterility controls were performed, as previously reported (Bertani et al. 2016). Plant material was finally macerated in phosphate-buffered saline (PBS) solution and serial dilutions of this macerate were plated in TSA containing the appropriate antibiotics, then incubated at $30^{\circ} \mathrm{C}$ for $24 \mathrm{~h}$ and counted for $\mathrm{CFU} / \mathrm{g}$ calculation.

In the case of rhizospheric colonization: roots were rinsed with sterile water removing all remaining Hoagland's semisolid solution and then vortexed in $5 \mathrm{ml}$ of PBS solution for $1 \mathrm{~min}$. Serial dilutions of this PBS solution were then plated on the appropriate selection media for calculation of CFU per gram.

Plasmid-loss calculation of Kosakonia plant colonization strains was performed by plating complemented Kosakonia cells KO348hcp(pBBRhcp) isolated from the rhizoplane and root endosphere in the following selective media: LB supplemented with $\mathrm{Km}$ at $100 \mu \mathrm{g} \mathrm{ml}{ }^{-1}$ plus $\mathrm{Gm}$ at $25 \mu \mathrm{g} \mathrm{ml}^{-1}$ for plasmid-complemented cells and LB supplemented with only $\mathrm{Km}$ at $100 \mu \mathrm{g} \mathrm{ml}^{-1}$ for cells which lost the plasmid. CFU per gram and percentage of plasmid loss was calculated.

For comparing the rhizoplane and endosphere colonization ability (Fig. 2) between KO348 and KO774 strains, a KruskallWallis test was used for specific pairs of data (KO348 versus KO774) in single and coinoculation by Prism 7 (Graphpad Software, Inc.). In the analysis of the rhizospheric and endophytic colonization of KO348 (Fig. 3) and on the effect of T6SS in colonization ability of KO348 (Fig. 6), a Kruskall-Wallis test was also used for corresponding specific pairs of data. All statistical analyses were performed with Prism 7 (Graphpad Software, Inc).

\section{Visualization of Kosakonia sp. KO348} in rice roots by confocal microscopy.

To further describe the colonization process by Kosakonia strains, rice plantlets were inoculated with strain KO348(pBBRgfp) 
harboring plasmid pBBR2GFP, which constitutively expressed the autofluorescent GFP protein (da Silva et al. 2014), as described above. Colonization assessment of rice by strain KO348 harboring the pBBR2GFP was performed at several time points $(5,10,30$, and $50 \mathrm{dpi}$ ). For surface visualization, samples (roots and shoots) taken from 10 plants at different time points were rinsed with distilled water and directly observed under a confocal microscope (Olympus Fluoview FV1000 with multiline laser FV5-LAMAR-2 $\mathrm{HeNe}(\mathrm{G})$ and laser FV10-LAHEG230-2). For internal colonization, samples were surface sterilized after being rinsed with $75 \%$ ethanol for 2 min and rinsed thrice with distilled water. Then, samples were treated with sodium hypochloride (7\%) solution for $2 \mathrm{~min}$ and rinsed, followed by two $75 \%$ ethanol treatments for $1 \mathrm{~min}$, and finally rinsed thrice with distilled water. Samples were then cut with a razor transversally or longitudinally and observed under the confocal microscope. $\mathrm{X}, \mathrm{Y}$, and $\mathrm{Z}$ pictures were taken at 405,488 , and $633 \mathrm{~nm}$, respectively, and with $\times 10, \times 20$, or $\times 40$ objectives. $Z$ stacks were observed using Imaris software or with Image J (National Institutes of Health, United States). Pictures were cropped and, due to the convolution process in the microscope, whole pictures were sharpened and the light/contrast balance improved to better observe the image details, as seen when samples are observed in the dark under the microscope (Glassner et al. 2015).

\section{Determination of the Kosakonia sp. KO348 protein secretome.}

In order to determine the proteins which were secreted by Kosakonia sp. KO348, the strain was grown in $200 \mathrm{ml}$ of plantmimicking AGF liquid media (Ryan et al. 2007) at $30^{\circ} \mathrm{C}$ for $16 \mathrm{~h}$ The culture was then centrifuged at $3,800 \times g$ at $4^{\circ} \mathrm{C}$ for $15 \mathrm{~min}$ and the spent supernatant was filtered through a 0.45 $\mu \mathrm{m}$ membrane in order to remove any residual bacterial cells. TCA was then added to a final concentration of $10 \%$ (wt/vol) and incubated for $16 \mathrm{~h}$ at $4^{\circ} \mathrm{C}$. Samples were then centrifuged for $60 \mathrm{~min}$ at $15,000 \times g$ at $4^{\circ} \mathrm{C}$. Pellets were washed with acetone and air dried. Protein pellets were then resuspended in NuPage 1× LDS buffer (Thermo Fisher Scientific Inc., Waltham, MA, U.S.A.), boiled for $5 \mathrm{~min}$, then run $3 \mathrm{~cm}$ in a precast NuPAGE $12 \%$ Bis-Tris gel (Thermo Fisher Scientific Inc.). The gels were stained with colloidal Coomassie brilliant blue (Sigma-Aldrich Inc., St. Louis, MS, U.S.A.). The stained area of the gel was cut into five bands and processed for in-gel digestion with trypsin using standard procedures (Wysocka et al. 2003). Liquid chromatography tandem mass spectrometry of the digests was performed using an Easy-nLC II coupled to an Amazon ETD mass spectrometer (Bruker Daltonics, Hamburg, Germany). The resulting spectra were searched using the X!tandem (The Global Protein Machine Organization) search engine and the Uniprot Kosakonia sp. KO348 published proteome (UP000253187) and filtered at a $2 \%$ false discovery rate. Table 1; Supplementary Tables S3 and S4 show the unique peptides and the statistical confidence of the protein matches.

\section{Construction of the Kosakonia strain KO348 hcp genomic knock-out mutant and its genetic complementation.}

A genomic knockout mutation of the hcp gene was constructed, using genomic DNA as template, by amplifying the 5' DNA flanking regions with primers pEXhcp1Fw 5'AGGATCCTTTAATTTCTACCCGCCTGG-3' and pEXhcp1Rv 5'-ACTCGAGTTTGCAGACAGACAGCTCAAC-3' and 3' DNA flanking regions with primers pEXhcp2Fw 5'-AGAATTCAGGTGTGACCTATGCATTCCA-3' and pEXhcp2Rv 5' -AGGTACCTTGTTTGACAGCCATTTCGG-3'. The 5' and 3' fragments were then ligated on either side of a $\mathrm{Km}$ resistance gene and the final fragment cloned in gene-replacement vector pEX19Gm (Hoang et al. 1998) generating pEX19Kmhcp. This latter plasmid was then electroporated into strain KO348 and, following selection $\left(\mathrm{Km}\right.$ resistant $\left[\mathrm{Km}^{\mathrm{R}}\right]$ and $\mathrm{Gm}$ susceptible $\left.\left[\mathrm{Gm}^{\mathrm{S}}\right]\right)$, resulted in the generation of an hcp knock-out mutant strain which was named Kosakonia KO348hcp.

The hcp full-length gene (including its gene promoter) was amplified with the primers prom+hcpFW $5^{\prime}$-AGGTACCTGTTTCTGAAGGTCGATGGAG- $3^{\prime}$ and prom+hcpRv 5'-AGGATCCTGTTTGACAGCCATTTCGGT-3', the sequence was verified via DNA sequencing, and the 802-bp fragment was cloned in the Gm-resistant $\left(\mathrm{Gm}^{\mathrm{R}}\right)$ pBBR1MCS-5 vector (Kovach et al. 1995). This plasmid was electroporated in the mutant strain KO348hcp and selected for $\mathrm{Km}^{\mathrm{R}}$ and $\mathrm{Gm}^{\mathrm{R}}$, and the resulting KO348hcp-complemented strain was named KO348hcp(pBBRhcp). The pBBR1MCS-5 plasmid vector (Kovach et al. 1995) was also electroporated in KO348hcp, resulting in KO348hcp(pBBRMCS-1).

\section{Bacterial growth curves.}

Three biological replicates of cultures of strains KO348, KO348hcp, and KO348hcp(pBBRhcp) were grown in LB broth supplemented with appropriate antibiotics and grown at $30^{\circ} \mathrm{C}$ with shaking at $200 \mathrm{rpm}$. $\mathrm{OD}_{600}$ values were measured every hour until reaching the stationary phase.

\section{Rice field trial using seed inoculated with a Kosakonia strain.}

A rice field trial using seed inoculated with Kosakonia sp. KO774 was carried out between May and October 2016 at Catarroja, Valencia, Spain $\left(39.3859292^{\circ} \mathrm{N}, 0.376225411^{\circ} \mathrm{W}\right)$. It consisted of 16 experimental plots of wet-seeded J. Sendra paddy rice divided into four groups of treatment as follows: (i) 8 plots received $100 \%$ nitrogen/urea recommended fertilization, with 4 of these planted with seed inoculated with Kosakonia sp. KO774 and the other 4 with seed not inoculated and (ii) 8 plots received $50 \%$ urea/nitrogen recommended fertilization, with 4 of these planted with seed inoculated with Kosakonia sp. KO774 and the other 4 with seed not inoculated. The seed inoculation with Kosakonia sp. KO774 was performed by soaking rice seed in a solution containing the strain at $10^{8}$ $\mathrm{CFU} / \mathrm{ml}$ for $24 \mathrm{~h}$. Rice plants were harvested at day 100 postrice-sowing and measurements, including germination/plot, weight (1,000 grains/plot and 25 panicles/plot), yield (per square meter), and yield (kilograms per hectare), were performed. Statistical analysis of variance was performed for analyzing the phenotypic differences between groups using Prism 7 (Graphpad Software, Inc).

\section{Microbiome studies.}

Microbiome analysis was performed on rice roots grown in two plots of the following two treatment groups of the field trials: (i) rice seed soaked in Kosakonia and the soil fertilized with $50 \%$ of the recommended nitrogen (treated group) and (ii) rice seed which were not inoculated with Kosakonia and the soil fertilized only with $50 \%$ of the recommended nitrogen (untreated group). Rice plants were collected at 30, 60, and 90 dpi and rice roots were washed and surface sterilized. In order to maintain the variability but decrease the number of samples, one sample was considered to be the sterilized roots of three different plants of rice derived from the same plot and collected at the same time point (Supplementary Fig. S7).

DNA from sterilized roots was extracted using PowerMax Soil DNA isolation kit (MO BIO Laboratories, Carlsbad, CA, U.S.A.) following the manufacturer's protocol and using $0.5 \mathrm{~g}$ of each sample. The $16 \mathrm{~S}$ rRNA gene amplicon library was prepared following the manufacturer's protocol (15044223 B; Illumina Inc., San Diego, CA, U.S.A.). Briefly, samples were amplified in the V3 and V4 regions using denaturated primers (Klindworth et al. 2013) in a limited-cycle PCR, followed by an 
AMPure XP bead clean-up (A638801; Beckman Coulter Inc., Brea, CA, U.S.A.). A second PCR was then performed to attach dual index and Illumina sequencing adapters using the Nextera XT Index Kit, followed by a final AMPure XP bead clean-up. 16S rRNA gene concentration was measured by fluorimetric quantification using Qubit 2 (Invitrogen Inc., Carlsbad, CA, U.S.A.). Sequencing was performed using the Illumina Miseq technology. The sequences of raw data were filtered out and the reads were trimmed to a consistent length. Then, the data were denoised, and chimera were filtered and taxonomically assigned using DADA2 v1.1.5 (Callahan et al. 2016). For the taxonomic analysis, the sequencing reads were clustered into operational taxonomic units, defined as groups of sequencing reads that differ by less than a fixed dissimilarity threshold (97\%) generated in DADA2 using the Greengeenes database v13.5 (The Greengenes Database Consortium) modified for including sequence "CTACGGGTGGCAGCAGTGGGGAA TTTTCCGCAATGGGCGAAAGCCTGACGGAGCAATGCC GCGTGGAGGTGGAAGGCCCACGGGTCGTCAACTTCTT TTCTCGGAGAAGAAACAATGACGGTATCTGAGGAATA AGCATCGGCTAACTCTGTGCCAGCAGCCGCGGTAAGA CAGAGGATGCAAGCGTTATCCGGAATGATTGGGCGTA AAGCGTCTGTAGGTGGCTTTTCAAGTCCGCCGTCAAA TCCCAGGGCTCAACCTGGGAACTGCATTCGAAACTGG CAGGCTGGAGTCTCGTAGAGGGAGGTAGAATTCCAGG TGTAGCGGTGAAATGCGTAGAGATCTGGAGGAATACC GGTGGCGAAGGCGGCCTCCTGGACGAAGACTGACGC TCAGGTGCGAAAGCGTGGGGAGCAAACAGGATTAGA TACCCCTGTAGT" as Bacteria Proteobacteria $\gamma$-Proteobacteria Enterobacterales Enterobacteriaceae Kosakonia_S (belonging to our strain KO774).

\section{ACKNOWLEDGMENTS}

We thank F. Moronta for preparing the genomic DNA and organizing the genome sequencing. The sequencing of strains of KO348 and KO774 was performed as part of Community Science Program Project 503198 of the Joint Genome Institute, Walnut Creek, CA, U.S.A

\section{LITERATURE CITED}

Alnajar, S., and Gupta, R. S. 2017. Phylogenomics and comparative genomic studies delineate six main clades within the family Enterobacteriaceae and support the reclassification of several polyphyletic members of the family. Infect. Genet. Evol. 54:108-127.

Baldani, J. I., Baldani, V. L. D., Seldin, L., and Dobereiner, J. 1986. Characterization of Herbaspirillum seropedicae gen. nov., sp. nov., a root-associated nitrogen-fixing bacterium. Int. J. Syst. Bacteriol. 36: 86-93.

Becker, M., Patz, S., Becker, Y., Berger, B., Drungowski, M., Bunk, B., Overmann, J., Spröer, C., Reetz, J., Tchakounte, G. V. T., and Ruppel, S. 1997. Comparative genomics reveal a flagellar system, a type VI secretion system and plant growth-promoting gene clusters unique to the endophytic bacterium Kosakonia radicincitans. Front. Microbiol. 9: 1997. doi:10.3389/fmicb.2018.01997

Berg, G. 2009. Plant-microbe interactions promoting plant growth and health: Perspectives for controlled use of microorganisms in agriculture. Appl. Microbiol. Biotechnol. 84:11-18.

Berg, G., Grube, M., Schloter, M., and Smalla, K. 2014. Unraveling the plant microbiome: Looking back and future perspectives. Front. Microbiol. 5:148.

Berg, G., Krechel, A., Ditz, M., Sikora, R. A., Ulrich, A., and Hallmann, J. 2005. Endophytic and ectophytic potato-associated bacterial communities differ in structure and antagonistic function against plant pathogenic fungi. FEMS Microbiol. Ecol. 51:215-229.

Berger, B., Brock, A. K., and Ruppel, S. 2013. Nitrogen supply influences plant growth and transcriptional responses induced by Enterobacter radicincitans in Solanum lycopersicum. Plant Soil 370:641-652.

Berger, B., Patz, S., Ruppel, S., Dietel, K., Faetke, S., Junge, H., and Becker, M. 2018. Successful formulation and application of plant growth-promoting Kosakonia radicincitans in maize cultivation. BioMed Res. Int. 2018:6439481. doi:10.1155/2018/6439481
Bergottini, V. M., Filippidou, S., Junier, T., Johnson, S., Chain, P. S., Otegui, M. B., Zapata, P. D., and Junier, P. 2015. Genome sequence of Kosakonia radicincitans strain YD4, a plant growth-promoting rhizobacterium isolated from yerba mate (Ilex paraguariensis St. Hill.). Genome Announce. 3:e00239-15.

Bernal, P., Llamas, M. A., and Filloux, A. 2018. Type VI secretion systems in plant-associated bacteria. Environ. Microbiol. 20:1-15.

Bertani, I., Abbruscato, P., Piffanelli, P., Subramoni, S., and Venturi, V. 2016. Rice bacterial endophytes: Isolation of a collection, identification of beneficial strains and microbiome analysis. Environ. Microbiol. Rep. 8:388-398.

Better, M., Lewis, B., Corbin, D., Ditta, G., and Helinski, D. R. 1983 Structural relationships among Rhizobium meliloti symbiotic promoters. Cell 35:479-485.

Boyer, F., Fichant, G., Berthod, J., Vandenbrouck, Y., and Attree, I. 2009. Dissecting the bacterial type VI secretion system by a genome wide in silico analysis: What can be learned from available microbial genomic resources? BMC Genomics 10:104.

Brader, G., Compant, S., Vescio, K., Mitter, B., Trognitz, F., Ma, L.-J., and Sessitsch, A. 2017. Ecology and genomic insights into plant-pathogenic and plant-nonpathogenic endophytes. Annu. Rev. Phytopathol. 55: 61-83.

Brady, C., Cleenwerck, I., Venter, S., Coutinho, T., and De Vos, P. 2013. Taxonomic evaluation of the genus Enterobacter based on multilocus sequence analysis (MLSA): Proposal to reclassify E. nimipressuralis and E. amnigenus into Lelliottia gen. nov. as Lelliottia nimipressuralis comb. nov. and Lelliottia amnigena comb. nov., respectively, E. gergoviae and E. pyrinus into Pluralibacter gen. nov. as Pluralibacter gergoviae' comb. nov. and Pluralibacter pyrinus comb. nov., respectively, E. cowanii, E. radicincitans, E. oryzae and E. arachidis into Kosakonia gen. nov. as Kosakonia cowanii comb. nov., Kosakonia radicincitans comb. nov., Kosakonia oryzae comb. nov. and Kosakonia arachidis comb. nov., respectively, and E. turicensis, E. helveticus and E. pulveris into Cronobacter as Cronobacter zurichensis nom. nov., Cronobacter helveticus comb. nov. and Cronobacter pulveris comb. nov., respectively, and emended description of the genera Enterobacter and Cronobacter. Syst. Appl. Microbiol. 36:309-319.

Bulgarelli, D., Garrido-Oter, R., Münch, P. C., Weiman, A., Dröge, J., Pan, Y., McHardy, A. C., and Schulze-Lefert, P. 2015. Structure and function of the bacterial root microbiota in wild and domesticated barley. Cell Host Microbe 17:392-403.

Bulgarelli, D., Rott, M., Schlaeppi, K., Ver Loren van Themaat, E., Ahmadinejad, N., Assenza, F., Rauf, P., Huettel, B., Reinhardt, R., Schmelzer, E., Peplies, J., Gloeckner, F. O., Amann, R., Eickhorst, T., and Schulze-Lefert, P. 2012. Revealing structure and assembly cues for Arabidopsis root-inhabiting bacterial microbiota. Nature 488:91-95.

Callahan, B. J., McMurdie, P. J., Rosen, M. J., Han, A. W., Johnson, A. J. A., and Holmes, S. P. 2016. DADA2: High-resolution sample inference from Illumina amplicon data. Nat. Methods 13:581-583.

Chaves, D. F. S., de Souza, E. M., Monteiro, R. A., and de Oliveira Pedrosa, F. 2009. A two-dimensional electrophoretic profile of the proteins secreted by Herbaspirillum seropedicae strain Z78. J. Proteomics 73: 50-56.

Chen, M., Zhu, B., Lin, L., Yang, L., Li, Y., and An, Q. 2014. Complete genome sequence of Kosakonia sacchari type strain SP1(T.). Stand. Genomic Sci. 9:1311-1318.

Compant, S., Clement, C., and Sessitsch, A. 2010. Plant growth-promoting bacteria in the rhizo- and endosphere of plants: Their role, colonization, mechanisms involved and prospects for utilization. Soil Biol. Biochem. 42:669-678.

Compant, S., Reiter, B., Sessitsch, A., Nowak, J., Clément, C., and Ait Barka, E. 2005. Endophytic colonization of Vitis vinifera L. by plant growth-promoting bacterium Burkholderia sp. strain PsJN. Appl. Environ. Microbiol. 71:1685-1693.

Coutinho, B. G., Mevers, E., Schaefer, A. L., Pelletier, D. A., Harwood, C. S., Clardy, J., and Greenberg, E. P. 2018. A plant-responsive bacterialsignaling system senses an ethanolamine derivative. Proc. Natl. Acad. Sci. U.S.A. 115:9785-9790.

da Silva, D. P., Castañeda-Ojeda, M. P., Moretti, C., Buonaurio, R., Ramos, C., and Venturi, V. 2014. Bacterial multispecies studies and microbiome analysis of a plant disease. Microbiology 160:556-566.

Divan Baldani, V. L., Baldani, J. I., and Döbereiner, J. 2000. Inoculation of rice plants with the endophytic diazotrophs Herbaspirillum seropedicae and Burkholderia spp. Biol. Fertil. Soils 30:485-491.

Edwards, J., Johnson, C., Santos-Medellín, C., Lurie, E., Podishetty, N. K. Bhatnagar, S., Eisen, J. A., and Sundaresan, V. 2015. Structure, variation, and assembly of the root-associated microbiomes of rice. Proc. Natl. Acad. Sci. U.S.A. 112:E911-E920. 
Edwards, J., Santos-Medellín, C., Liechty, Z., Nguyen, B., Lurie, E., and Ave, S. 2017. Compositional shifts in the root microbiota track the lifecycle of field-grown rice plants. bioRxiv 166025. doi:10.1101/166025

Egener, T., Hurek, T., and Reinhold-Hurek, B. 1999. Endophytic expression of nif genes of Azoarcus sp. strain BH72 in rice roots. Mol. PlantMicrobe Interact. 12:813-819.

FAO. 2017. World Fertilizer Trends and Outlook to 2020. Food and Agriculture Organization of the United Nations, Rome, Italy. http:// www.fao.org/3/a-i6895e.pdf

Feng, Y., Shen, D., and Song, W. 2006. Rice endophyte Pantoea agglomerans YS19 promotes host plant growth and affects allocations of host photosynthates. J. Appl. Microbiol. 100:938-945.

Fox, A., Kwapinski, W., Griffiths, B. S., and Schmalenberger, A. 2014. The role of sulfur- and phosphorus-mobilizing bacteria in biochar-induced growth promotion of Lolium perenne. FEMS Microbiol. Ecol. 90:78-91.

Frank, A. C. 2011. The genomes of endophytic bacteria. Pages 107-136 in: Endophytes of Forest Trees. Forestry Sciences, vol. 80. A. Pirttilä and A. Frank, eds. Springer, Dordrecht, The Netherlands.

Garrido-Oter, R., Nakano, R. T., Dombrowski, N., Ma, K.-W., The AgBiome Team4, McHardy, A. C., and Schulze-Lefert, P. 2018. Modular traits of the rhizobiales root microbiota and their evolutionary relationship with symbiotic rhizobia. Cell Host Microbe 24:155-167.e5.

Glassner, H., Zchori-Fein, E., Compant, S., Sessitsch, A., Katzir, N., Portnoy, V., and Yaron, S. 2015. Characterization of endophytic bacteria from cucurbit fruits with potential benefits to agriculture in melons (Cucumis melo L.). FEMS Microbiol. Ecol. 91:fiv074. doi:10.1093/femsec/fiv074

Glick, B. R. 2014. Bacteria with ACC deaminase can promote plant growth and help to feed the world. Microbiol. Res. 169:30-39.

Govindarajan, M., Balandreau, J., Kwon, S. W., Weon, H. Y., and Lakshminarasimhan, C. 2008. Effects of the inoculation of Burkholderia vietnamensis and related endophytic diazotrophic bacteria on grain yield of rice. Microb. Ecol. 55:21-37.

Gupta, S., and Dikshit, A. K. 2010. Biopesticides: An ecofriendly approach for pest control. J. Biopestic. 3:186-188.

Gyaneshwar, P., James, E. K., Mathan, N., Reddy, P. M., Reinhold-Hurek, B., and Ladha, J. K. 2001. Endophytic colonization of rice by a diazotrophic strain of Serratia marcescens. J. Bacteriol. 183:2634-2645.

Hardoim, P. R. 2015. Heading to the origins-Rice microbiome as functional extension of the host. J. Rice Res. 3:133. doi:10.4172/2375-4338.1000133

Hartmann, A., Rothballer, M., and Schmid, M. 2008. Lorenz Hiltner, a pioneer in rhizosphere microbial ecology and soil bacteriology research. Plant Soil 312:7-14.

Hayat, R., Ali, S., Amara, U., Khalid, R., and Ahmed, I. 2010. Soil beneficial bacteria and their role in plant growth promotion: A review. Ann. Microbiol. 60:579-598.

Hoang, T. T., Karkhoff-Schweizer, R. R., Kutchma, A. J., and Schweizer, H. P. 1998. A broad-host-range Flp-FRT recombination system for sitespecific excision of chromosomally-located DNA sequences: Application for isolation of unmarked Pseudomonas aeruginosa mutants. Gene 212:77-86.

Höflich, G., and Ruppel, S. 1994. Growth stimulation of pea after inoculation with associative bacteria. Microbiol. Res. 149:99-104.

Hurek, T., Reinhold-Hurek, B., Van Montagu, M., and Kellenberger, E. 1994. Root colonization and systemic spreading of Azoarcus sp. strain BH72 in grasses. J. Bacteriol. 176:1913-1923.

Jiang, J., Wu, S., Wang, J., and Feng, Y. 2015. AHL-type quorum sensing and its regulation on symplasmata formation in Pantoea agglomerans YS19. J. Basic Microbiol. 55:607-616.

Kamat, S. S., and Raushel, F. M. 2013. The enzymatic conversion of phosphonates to phosphate by bacteria. Curr. Opin. Chem. Biol. 17:589-596.

Kämpfer, P., McInroy, J. A., Doijad, S., Chakraborty, T., and Glaeser, S. P. 2016. Kosakonia pseudosacchari sp. nov., an endophyte of Zea mays. Syst. Appl. Microbiol. 39:1-7.

Kaval, K. G., and Garsin, D. A. 2018. Ethanolamine utilization in bacteria. MBio 9:e00066-18.

Khush, G. 2003. Productivity improvements in rice. Nutr. Rev. 61: S114-S116.

Klindworth, A., Pruesse, E., Schweer, T., Peplies, J., Quast, C., Horn, M., and Glöckner, F. O. 2013. Evaluation of general 16S ribosomal RNA gene PCR primers for classical and next-generation sequencing-based diversity studies. Nucleic Acids Res. 41:e1.

Kovach, M. E., Elzer, P. H., Hill, D. S., Robertson, G. T., Farris, M. A., Roop, R. M., 2nd, and Peterson, K. M. 1995. Four new derivatives of the broad-host-range cloning vector pBBR1MCS, carrying different antibiotic-resistance cassettes. Gene 166:175-176.

Ladha, J., Tirol-Padre, A., Punzalan, G., and Watanabe, I. 1987. Nitrogenfixing $\left(\mathrm{C}_{2} \mathrm{H}_{2}\right.$-reducing $)$ activity and plant growth characters of 16 wetland rice varieties. Soil Sci. Plant Nutr. 33:187-200.
Levy, A., Salas Gonzalez, I., Mittelviefhaus, M., Clingenpeel, S., Herrera Paredes, S., Miao, J., Wang, K., Devescovi, G., Stillman, K., Monteiro, F., Rangel Alvarez, B., Lundberg, D. S., Lu, T. Y., Lebeis, S., Jin, Z., McDonald, M., Klein, A. P., Feltcher, M. E., Rio, T. G., Grant, S. R., Doty, S. L., Ley, R. E., Zhao, B., Venturi, V., Pelletier, D. A., Vorholt, J. A., Tringe, S. G., Woyke, T., and Dangl, J. L. 2018. Genomic features of bacterial adaptation to plants. Nat. Genet. 50:138-150.

Li, Y., Li, S., Chen, M., Peng, G., Tan, Z., and An, Q. 2017. Complete genome sequence of Kosakonia oryzae type strain Ola $51^{\mathrm{T}}$. Stand. Genomic Sci. 12:28

Lòpez-Fernàndez, S., Mazzoni, V., Pedrazzoli, F., Pertot, I., and Campisano, A. 2017. A phloem-feeding insect transfers bacterial endophytic communities between grapevine plants. Front. Microbiol. 8:834.

Lugtenberg, B., and Kamilova, F. 2009. Plant-growth-promoting rhizobacteria. Annu. Rev. Microbiol. 63:541-556.

Luna, M. F., Galar, M. L., Aprea, J., Molinari, M. L., and Boiardi, J. L. 2010. Colonization of sorghum and wheat by seed inoculation with Gluconacetobacter diazotrophicus. Biotechnol. Lett. 32 1071-1076.

Lundberg, D. S., Lebeis, S. L., Paredes, S. H., Yourstone, S., Gehring, J., Malfatti, S., Tremblay, J., Engelbrektson, A., Kunin, V., Del Rio, T. G., Edgar, R. C., Eickhorst, T., Ley, R. E., Hugenholtz, P., Tringe, S. G., and Dangl, J. L. 2012. Defining the core Arabidopsis thaliana root microbiome. Nature 488:86-90.

Ma, Y., Rajkumar, M., Luo, Y., and Freitas, H. 2011. Inoculation of endophytic bacteria on host and non-host plants-Effects on plant growth and Ni uptake. J. Hazard. Mater. 195:230-237.

Mahanty, T., Bhattacharjee, S., Goswami, M., Bhattacharyya, P., Das, B., Ghosh, A., and Tribedi, P. 2017. Biofertilizers: A potential approach for sustainable agriculture development. Environ. Sci. Pollut. Res. Int. 24: 3315-3335.

Mano, H., and Morisaki, H. 2008. Endophytic bacteria in the rice plant. Microbes Environ. 23:109-117.

Meng, X., Bertani, I., Abbruscato, P., Piffanelli, P., Licastro, D., Wang, C., and Venturi, V. 2015. Draft genome sequence of rice endophyteassociated isolate Kosakonia oryzae KO348. Genome Announce. 3 : e00594-15.

Mohd Suhaimi, N. S., Yap, K.-P., Ajam, N., and Thong, K.-L. 2014. Genome sequence of Kosakonia radicincitans UMEnt01/12, a bacterium associated with bacterial wilt diseased banana plant. FEMS Microbiol. Lett. 358:11-13.

Müller, D. B., Vogel, C., Bai, Y., and Vorholt, J. A. 2016. The plant microbiota: Systems-level insights and perspectives. Annu. Rev. Genet. 50:211-234.

Nordberg, H., Cantor, M., Dusheyko, S., Hua, S., Poliakov, A., Shabalov, I., Smirnova, T., Grigoriev, I. V., and Dubchak, I. 2014. The genome portal of the Department of Energy Joint Genome Institute: 2014 updates. Nucleic Acids Res. 42:D26-D31.

Okubo, T., Ikeda, S., Sasaki, K., Ohshima, K., Hattori, M., Sato, T., and Minamisawa, K. 2014. Phylogeny and functions of bacterial communities associated with field-grown rice shoots. Microbes Environ. 29: 329-332.

Pham, V. T. K., Rediers, H., Ghequire, M. G. K., Nguyen, H. H., De Mot, R., Vanderleyden, J., and Spaepen, S. 2017. The plant growth-promoting effect of the nitrogen-fixing endophyte Pseudomonas stutzeri A15. Arch. Microbiol. 199:513-517.

Reinhold-Hurek, B., and Hurek, T. 1998. Life in grasses: Diazotrophic endophytes. Trends Microbiol. 6:139-144.

Reinhold-Hurek, B., and Hurek, T. 2011. Living inside plants: Bacterial endophytes. Curr. Opin. Plant Biol. 14:435-443.

Rouws, L. F. M., Meneses, C. H. S. G., Guedes, H. V., Vidal, M. S., Baldani, J. I., and Schwab, S. 2010. Monitoring the colonization of sugarcane and rice plants by the endophytic diazotrophic bacterium Gluconacetobacter diazotrophicus marked with gfp and gusA reporter genes. Lett. Appl. Microbiol. 51:325-330.

Ryan, R. P., Fouhy, Y., Lucey, J. F., Jiang, B. L., He, Y. Q., Feng, J. X., Tang, J. L., and Dow, J. M. 2007. Cyclic di-GMP signalling in the virulence and environmental adaptation of Xanthomonas campestris. Mol. Microbiol. 63:429-442.

Saikia, S. P., and Jain, V. 2007. Biological nitrogen fixation with nonlegumes: An achievable target or a dogma? Curr. Sci. 92:317-322.

Santi, C., Bogusz, D., and Franche, C. 2013. Biological nitrogen fixation in non-legume plants. Ann. Bot. 111:743-767.

Schlaeppi, K., and Bulgarelli, D. 2015. The plant microbiome at work. Mol. Plant-Microbe Interact. 28:212-217.

Schmidt, M. A., Souza, E. M., Baura, V., Wassem, R., Yates, M. G., Pedrosa, F. O., and Monteiro, R. A. 2011. Evidence for the endophytic 
colonization of Phaseolus vulgaris (common bean) roots by the diazotroph Herbaspirillum seropedicae. Braz. J. Med. Biol. Res. 44: 182-185.

Schreiner, M., Krumbein, A., and Ruppel, S. 2009. Interaction between plants and bacteria: Glucosinolates and phyllospheric colonization of cruciferous vegetables by Enterobacter radicincitans DSM 16656. J. Mol. Microbiol. Biotechnol. 17:124-135.

Schütz, L., Gattinger, A., Meier, M., Müller, A., Boller, T., Mäder, P., and Mathimaran, N. 2018. Improving crop yield and nutrient use efficiency via biofertilization-A global meta-analysis. Front. Plant Sci. 8:2204.

Sessitsch, A., Hardoim, P., Döring, J., Weilharter, A., Krause, A., Woyke, T., Mitter, B., Hauberg-Lotte, L., Friedrich, F., Rahalkar, M., Hurek, T., Sarkar, A., Bodrossy, L., van Overbeek, L., Brar, D., van Elsas, J. D., and Reinhold-Hurek, B. 2012. Functional characteristics of an endophyte community colonizing rice roots as revealed by metagenomic analysis. Mol. Plant-Microbe Interact. 25:28-36.

Shinjo, R., Uesaka, K., Ihara, K., Loshakova, K., Mizuno, Y., Yano, K., and Tanaka, A. 2016. Complete genome sequence of Kosakonia sacchari strain BO-1, an endophytic diazotroph isolated from a sweet potato. Genome Announce. 4:e00868-16.

Steindler, L., Bertani, I., De Sordi, L., Schwager, S., Eberl, L., and Venturi, V. 2009. LasI/R and RhlI/R quorum sensing in a strain of Pseudomonas aeruginosa beneficial to plants. Appl. Environ. Microbiol. 75:51315140.

Sun, S., Chen, Y., Cheng, J., Li, Q., Zhang, Z., and Lan, Z. 2018. Isolation, characterization, genomic sequencing, and GFP-marked insertional mutagenesis of a high-performance nitrogen-fixing bacterium, Kosakonia radicincitans GXGL-4A and visualization of bacterial colonization on cucumber roots. Folia Microbiol. (Praha) 63:789-802.

Taga, M. E., and Walker, G. C. 2010. Sinorhizobium meliloti requires a cobalamin-dependent ribonucleotide reductase for symbiosis with its plant host. Mol. Plant-Microbe Interact. 23:1643-1654.

Taghavi, S., Wu, X., Ouyang, L., Zhang, Y. B., Stadler, A., McCorkle, S., Zhu, W., Maslov, S., and van der Lelie, D. 2015. Transcriptional responses to sucrose mimic the plant-associated life style of the plant growth promoting endophyte Enterobacter sp. 638. PLoS One 10: e0115455.

Trân Van, V., Berge, O., Balandreau, J., Ngô Ké, S., and Heulin, T. 1996. Isolement et activité nitrogénasique de Burkholderia vietnamiensis, bactérie fixatrice d'azote associée au riz (Oryza sativa L) cultivé sur un sol sulfaté du Viêt-nam. Agronomie 16:479-491. doi:10.1051/agro:19960802

Turner, T. R., James, E. K., and Poole, P. S. 2013. The plant microbiome. Genome Biol. 14:209.

Van Brussel, A. A., Zaat, S. A., Cremers, H. C., Wijffelman, C. A., Pees, E., Tak, T., and Lugtenberg, B. J. 1986. Role of plant root exudate and Sym plasmid-localized nodulation genes in the synthesis by Rhizobium leguminosarum of Tsr factor, which causes thick and short roots on common vetch. J. Bacteriol. 165:517-522.

van der Heijden, M. G. A., and Hartmann, M. 2016. Networking in the plant microbiome. PLoS Biol. 14:e1002378.

Varghese, N. J., Mukherjee, S., Ivanova, N., Konstantinidis, K. T., Mavrommatis, K., Kyrpides, N. C., and Pati, A. 2015. Microbial species delineation using whole genome sequences. Nucleic Acids Res. 43: 6761-6771.

Walitang, D. I., Kim, C.-G., Jeon, S., Kang, Y., and Sa, T. 2019. Conservation and transmission of seed bacterial endophytes across generations following crossbreeding and repeated inbreeding of rice at different geographic locations. MicrobiologyOpen 8:e00662. doi: $10.1002 / \mathrm{mbo} 3.662$

Walitang, D. I., Kim, C. G., Kim, K., Kang, Y., Kim, Y. K., and Sa, T. 2018. The influence of host genotype and salt stress on the seed endophytic community of salt-sensitive and salt-tolerant rice cultivars. BMC Plant Biol. 18:51.

Walitang, D. I., Kim, K., Madhaiyan, M., Kim, Y. K., Kang, Y., and Sa, T. 2017. Characterizing endophytic competence and plant growth promotion of bacterial endophytes inhabiting the seed endosphere of rice. BMC Microbiol. 17:209. doi:10.1186/s12866-017-1117-0

Witzel, K., Gwinn-Giglio, M., Nadendla, S., Shefchek, K., and Ruppel, S. 2012. Genome sequence of Enterobacter radicincitans DSM16656(T), a plant growth-promoting endophyte. J. Bacteriol. 194:5469.

Wysocka, J., Myers, M. P., Laherty, C. D., Eisenman, R. N., and Herr, W. 2003. Human Sin3 deacetylase and trithorax-related Set1/Ash2 histone H3-K4 methyltransferase are tethered together selectively by the cellproliferation factor HCF-1. Genes Dev. 17:896-911.

Yang, H., Sun, X., Song, W., Wang, Y., and Cai, M. 1999. Screening, identification and distribution of endophytic associative diazotrophs isolated from rice plants. (Abstr.) Acta Bot. Sin. 41:927-931.

Zeigler, R. S., and Barclay, A. 2008. The relevance of rice. Rice 1:3-10. 